\title{
ESTIMACIÓN DE ESPECTROS DE ACELERACIONES CORRESPONDIENTES A DIFERENTES PERIODOS DE RETORNO PARA LAS DISTINTAS ZONAS SÍSMICAS DE LA CIUDAD DE MÉXICO
}

\author{
Carlos Reyes $^{(1)}$, Eduardo Miranda (2), Mario Ordaz ${ }^{(3)}$ \\ y Roberto Meli $^{(3)}$
}

\begin{abstract}
RESUMEN
Se presenta una comparación entre dos procedimientos, forma espectral normalizada y ley de atenuación espectral, para estimar el espectro de aceleraciones horizontales absolutas para una fracción del 5 por ciento del amortiguamiento crítico en la estación acelerográfica CU. Esta estación se localiza en la zona de terreno firme de la ciudad de México, específicamente en el Instituto de Ingeniería de la Universidad Nacional Autónoma de México. De la comparación, resulta que la ley de atenuación espectral es el mejor procedimiento para estimar el espectro de aceleraciones en la estación CU. También, se estudia la técnica que se basa en los cocientes espectrales de respuesta (CER) para estimar el espectro de aceleraciones en las estaciones de la zona de terreno blando de la ciudad. Del análisis de resultados, se concluye que la mayor fuente de incertidumbre se debe a la estimación del espectro en la estación CU. Finalmente, se presentan ejemplos de espectros de peligro uniforme para distintos periodos de retorno en la estación $\mathrm{CU}$ y en estaciones localizadas en la zona de terreno blando de la ciudad.
\end{abstract}

\section{SUMMARY}

A comparison between two procedures, normalized spectral shape and spectral attenuation law, to estimate the acceleration response spectra in the CU station is presented. The CU station is located in the Institute of Engineering of the National Autonomous University of Mexico, in Mexico City. Comparison of the results of both methods indicates that the spectral attenuation law is the better procedure to estimate the acceleration response spectral in that station. Also, a technique based on response spectral ratios to estimate the acceleration response spectra in the soft soil zone of Mexico City is studied. This study concludes that the major source of uncertainty is the response spectra in the CU station. Finally, examples of uniform hazard spectra in the station CU and the soft soil zone of Mexico City are presented.

Artículo recibido el 8 de junio de 2001 y aprobado para su publicación el 26 de marzo de 2002. Se aceptarán comentarios y/o discusiones hasta cinco meses después de su publicación.

(1) Centro Nacional de Prevención de Desastres, Delfín Madrigal No.665, 04360 México, DF.creyes@cenapred.unam.mx

(2) Department of Civil and Environmental Engineering, Stanford University, miranda@cive.stanford.edu

(3) Instituto de Ingeniería, UNAM, Ciudad Universitaria, 04510 México, DF., mors@pumas.iingen.unam.mx, rmep@pumas.iingen.unam.mx 


\section{INTRODUCCIÓN}

Una de las herramientas más útiles para propósitos de ingeniería sísmica es el espectro de respuesta, el cual se usó por primera vez por Benioff (1934), posteriormente por Housner (1941) y Biot en el mismo año. Actualmente, el espectro de respuesta es la base en la mayoría de los reglamentos de diseño sísmico en el mundo para establecer las fuerzas de diseño con las que se proporciona la rigidez y resistencia a las estructuras. El espectro de respuesta elástico para un registro de movimiento de terreno caracterizado por una historia de aceleraciones, representa la respuesta máxima de una serie de osciladores de un grado de libertad con periodo y amortiguamiento determinados. Los tipos de espectros de respuesta elástica pueden ser una aceleración absoluta, una velocidad relativa o bien un desplazamiento relativo.

En estudios de peligro y riesgo sísmico el interés se centra en estimar el espectro de respuesta para temblores futuros; para hacerlo se han desarrollado distintos procedimientos, entre ellos destacan los que se mencionan a continuación.

\section{Escalar una forma espectral normalizada}

Para obtener un espectro de respuesta con este procedimiento se escala una forma espectral normalizada, que es el resultado de promediar varias de estas formas correspondientes a los distintos sismos que se han registrado en un sitio determinado. El factor de normalización y escalamiento, generalmente es la aceleración máxima del terreno. En la fig.1 se muestra esquemáticamente el procedimiento, que en resumen consiste en multiplicar una forma espectral normalizada por un cierto nivel de aceleración máxima del terreno para obtener un estimado del espectro de aceleraciones. En la figura $\mathrm{Sa}$ es la aceleración espectral, $\mathrm{a}_{\operatorname{máx}}$ es la aceleración máxima del terreno, $\mathrm{T}$ es el periodo de un oscilador de un grado de libertad, $\mathrm{R}$ es la distancia epicentral y $\mathrm{M}_{1}, \mathrm{M}_{2}$ y $\mathrm{M}_{3}$ son tres magnitudes para las cuales se obtiene la variación de $\mathrm{a}_{\text {máx }}$ con la distancia.

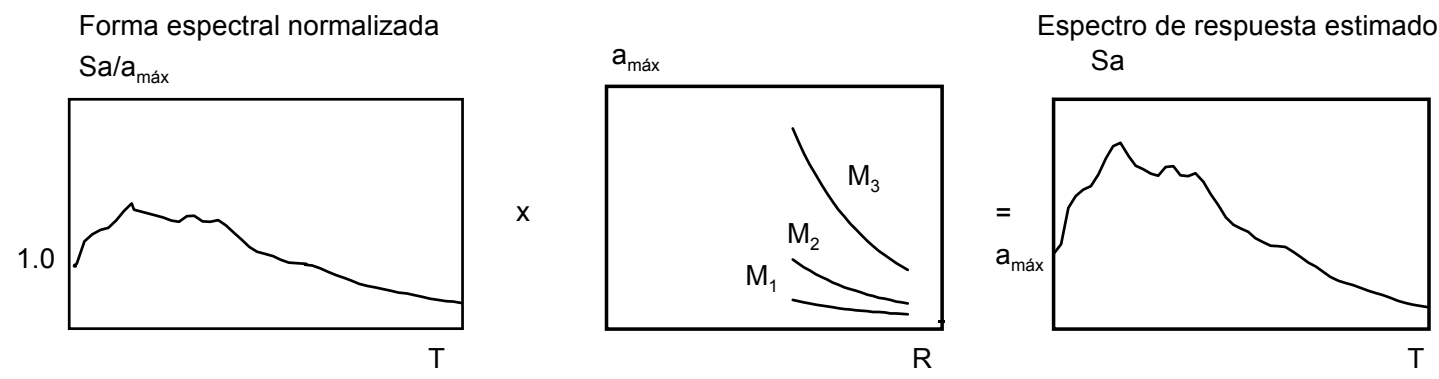

Figura 1. Procedimiento para estimar el espectro de respuesta usando la forma espectral normalizada.

Las primeras formas espectrales fueron desarrolladas por Housner (1959), quien las obtuvo para ocho registros de suelo firme correspondientes a los componentes EW y NS de cuatro sismos ocurridos en Estados Unidos. En estudios posteriores Hayashi et al. (1971) y Seed et al. (1976), obtuvieron formas espectrales normalizadas asociadas a distintos tipos de suelo. 
En lo que se refiere al factor de escalamiento, se obtiene a través de leyes de atenuación, que sirven para estimar movimientos máximos del terreno, es decir, aceleración, velocidad o desplazamiento. Las primeras leyes de atenuación se comenzaron a desarrollar en el siglo pasado, en la década de los años sesenta, por Kanai (1961) y Esteva (1968). Ésta última después fue modificada en una publicación de Esteva y Villaverde (1974). Cabe mencionar que las leyes de atenuación publicadas en 1968 y 1974, aunque fueron desarrolladas en México, en ellas se empleó un porcentaje elevado de datos de temblores ocurridos en Estados Unidos. Dentro de las leyes de atenuación en las que se emplean únicamente datos de temblores ocurridos en México, destacan las de Bufaliza (1984), Singh, Mena y Castro (1988) y Ordaz, Jara y Singh (1989).

\section{Ley de atenuación espectral}

Las leyes de atenuación espectrales son una extensión a varios periodos de las leyes de atenuación para estimar movimientos máximos del terreno. Esto implica, calcular, a través de una regresión, una serie de coeficientes para cada periodo considerado y de acuerdo al funcional que se use con el fin de describir el espectro de respuesta.

El desarrollo de las leyes de atenuación espectrales comenzó en la década de los años setenta en Estados Unidos en estudios hechos por McGuire (1974) y Trifunac y Anderson (1978); mas recientemente se puede mencionar el trabajo realizado por Joyner y Boore (1988) . En México, destaca el trabajo de Perea y Sordo (1998), aplicable a la ciudad de Puebla.

\section{Uso del espectro de amplitudes de Fourier}

Con esta técnica, el espectro de respuesta se estima a partir del espectro de amplitudes de Fourier y de la teoría de vibraciones aleatorias. Dicha teoría, toma al espectro de amplitudes de Fourier como un estimador de la densidad espectral y, junto con una estimación de la duración de la respuesta de un oscilador de un grado de libertad sometido a una excitación sísmica en su base, permite estimar las ordenadas del espectro de respuesta.

Los pioneros en el desarrollo de este procedimiento fueron Cartwright y Longuett-Higgins (1956), Davenport (1964) y Udwadia y Trifunac (1974). En México, la primera aplicación fue el trabajo de Ordaz y Reinoso (1987).

De los tres procedimientos anteriores, los dos últimos (ley de atenuación espectral y espectro de amplitudes de Fourier) tienen en cuenta la variación de la forma del espectro de respuesta con la magnitud y la distancia, mientras que el primero (forma espectral normalizada) no tiene en cuenta en forma explícita el efecto de ninguno de estos dos parámetros.

\section{Estimación del espectro de respuesta en suelos blandos}

Para fines de ingeniería sísmica el suelo de la ciudad de México se divide en tres zonas: la zona I que corresponde a la zona de terreno firme, la zona II llamada zona de transición, y la zona III, 
que corresponde a la antigua zona del lago y en la que existen mantos de arcilla de más de $20 \mathrm{~m}$ de profundidad. En suelos blandos, como los que existen en la zona transición y la zona del lago de la ciudad de México, debido a su característica de comportamiento prácticamente lineal, desde hace algunos años se ha utilizado la técnica de los cocientes espectrales con el fin de estimar el espectro de respuesta. El cociente espectral es la relación entre un espectro de zona blanda y uno de zona firme; representa una estimación de la amplificación de la intensidad espectral en terreno blando respecto a la del terreno firme. Un cociente espectral, se puede calcular usando tanto el espectro de Fourier como el de respuesta; en el caso del espectro de amplitudes de Fourier, dicho cociente es una función de transferencia empírica, mientras que en el caso del espectro de respuesta no tiene significado físico. Aún con esta deficiencia y considerando también, que el procedimiento tiene limitaciones por suponer, implícitamente, un modelo de amplificación del movimiento del suelo unidimensional, los resultados que se obtienen con el cociente de espectros de respuesta tienen una aproximación semejante a la que se obtiene de usar el espectro de amplitudes de Fourier (Rosenblueth y Arciniega, 1992). En la fig. 2, se muestran esquemáticamente los dos procedimientos para estimar espectros de respuesta a partir de la estimación del movimiento en zona firme. El emplear el espectro de amplitudes de Fourier para estimar el de respuesta demanda una mayor cantidad de cálculos numéricos; asimismo, se requiere de conocimientos en el tema de vibraciones aleatorias. Emplear directamente el espectro de respuesta en terreno firme y los cocientes espectrales de respuesta (CER), es una técnica más sencilla que demanda menor cantidad de cálculos numéricos; por tal motivo es el procedimiento que se utiliza en el presente trabajo para estimar el espectro de respuesta en suelos blandos.

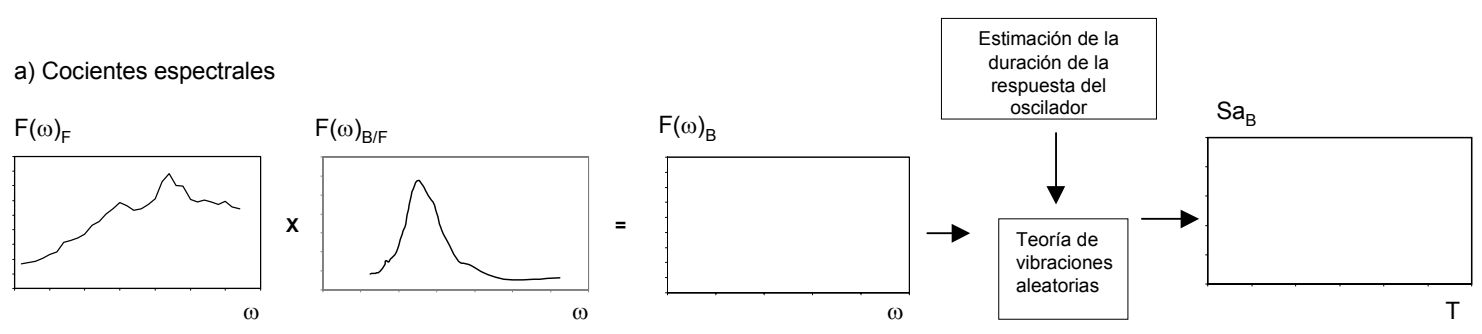

b) Cocientes espectrales de respuesta

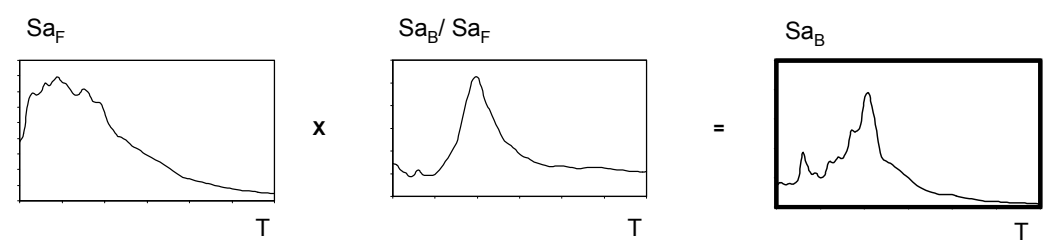

Figura 2. Procedimientos para estimar el espectro de respuesta en las zonas de transición y del lago a partir de la estimación del movimiento en suelo firme.

Después del sismo del 19 de septiembre de 1985, se colocaron más de 100 acelerógrafos en la ciudad de México, los cuales están localizados en los diferentes tipos de suelo. La instrumentación ha permitido estimar espectros de respuesta en la zona de transición y del lago, usando los CER y el espectro de respuesta en la estación CU. La estación CU, es la estación con mayor antigüedad en la ciudad de México, operando desde 1965; esto permite reducir sustancialmente las incertidumbres al estimar el espectro de respuesta en esa estación, para después usarlo como base y así poder estimar espectros en las estaciones de suelo blando. 


\section{OBJETIVOS DEL ESTUDIO}

En la primera parte del trabajo, el objetivo es comparar los dos primeros procedimientos citados en la sección anterior, forma espectral normalizada y ley de atenuación espectral, para estimar el espectro de aceleraciones absolutas considerando una fracción igual al 5\% de amortiguamiento crítico en la estación CU. Esta comparación permite establecer cuál de los dos procedimientos es el más adecuado para estimar el espectro de aceleraciones en la estación en cuestión.

En una segunda parte del trabajo, el objetivo es estudiar el error que se comete al emplear los CER para estimar espectros de respuesta en la zonas de transición y del lago. Finalmente, con el uso de curvas teóricas de peligro sísmico se obtienen espectros de aceleraciones con diferentes periodos de retorno en la estación CU y en algunas estaciones de las zonas de transición y del lago.

\section{ESTIMACIÓN DEL ESPECTRO DE RESPUESTA EN LA ESTACIÓN CU PARA UN 5\% DEL AMORTIGUAMIENTO CRÍTICO}

\section{Datos utilizados}

Los registros que se tomaron para este estudio son los correspondientes a sismos de subducción con magnitud $\mathrm{M}_{\mathrm{w}}>6.0$ y epicentros localizados en la costa del Pacífico mexicano entre el 23 de agosto de 1965 y el 14 de septiembre de 1995. Durante este periodo se registraron en la estación CU, 17 temblores con magnitud $\mathrm{M}_{\mathrm{w}}>6.0$. Respecto a los eventos que se tienen en cuenta en este estudio se hacen las siguientes observaciones:

1. Los registros que se utilizan corresponden a sismos con distancias epicentrales comprendidas entre 280 y $466 \mathrm{~km}$, y magnitudes $\left(\mathrm{M}_{\mathrm{w}}\right)$ entre 6.1 y 8.1. Estos datos se obtuvieron de Ordaz et al. (1994) y del catalogo de sismos proporcionado por Zúñiga (1996);

2. Para los sismos de 1985 se tomaron en cuenta dos estaciones adicionales. La primera de ellas llamada estación cuip (Instituto de Ingeniería patio), y la segunda llamada estación cumv (se ubica en la mesa vibradora del Instituto de Ingeniería). Ambas estaciones se localizan a menos de $2 \mathrm{~km}$ de la estación $\mathrm{CU}$, por esta razón se considera que son representativas del movimiento en esta estación, además de enriquecer los datos disponibles.

En los dos procedimientos que se estudian para estimar el espectro de respuesta en la estación CU, se tienen en cuenta los componentes del movimiento del terreno EW, NS y la media geométrica de la aceleración espectral de ambos componentes del movimiento (MG), definida como:

$\mathrm{Sa}_{\mathrm{MG}}=\left[\left(\mathrm{Sa}_{\mathrm{EW}}{ }^{2}+\mathrm{Sa}_{\mathrm{NS}}{ }^{2}\right) / 2\right]^{1 / 2}$

Los espectros de los sismos considerados se muestran en la fig. 3; el ccomponente EW con línea continua, el componente NS con línea discontinua y la media geométrica de la aceleración espectral de los dos componentes horizontales del movimiento con línea punteada. Se aprecia que 
los espectros presentan un pico entre 1 y 2 segundos y en otros casos dos picos, mostrando que la forma del espectro en la estación CU depende de la magnitud del sismo o bien que el suelo donde se localiza esta estación presenta cierta amplificación; como se muestra en el trabajo de Ordaz y Singh (1992).
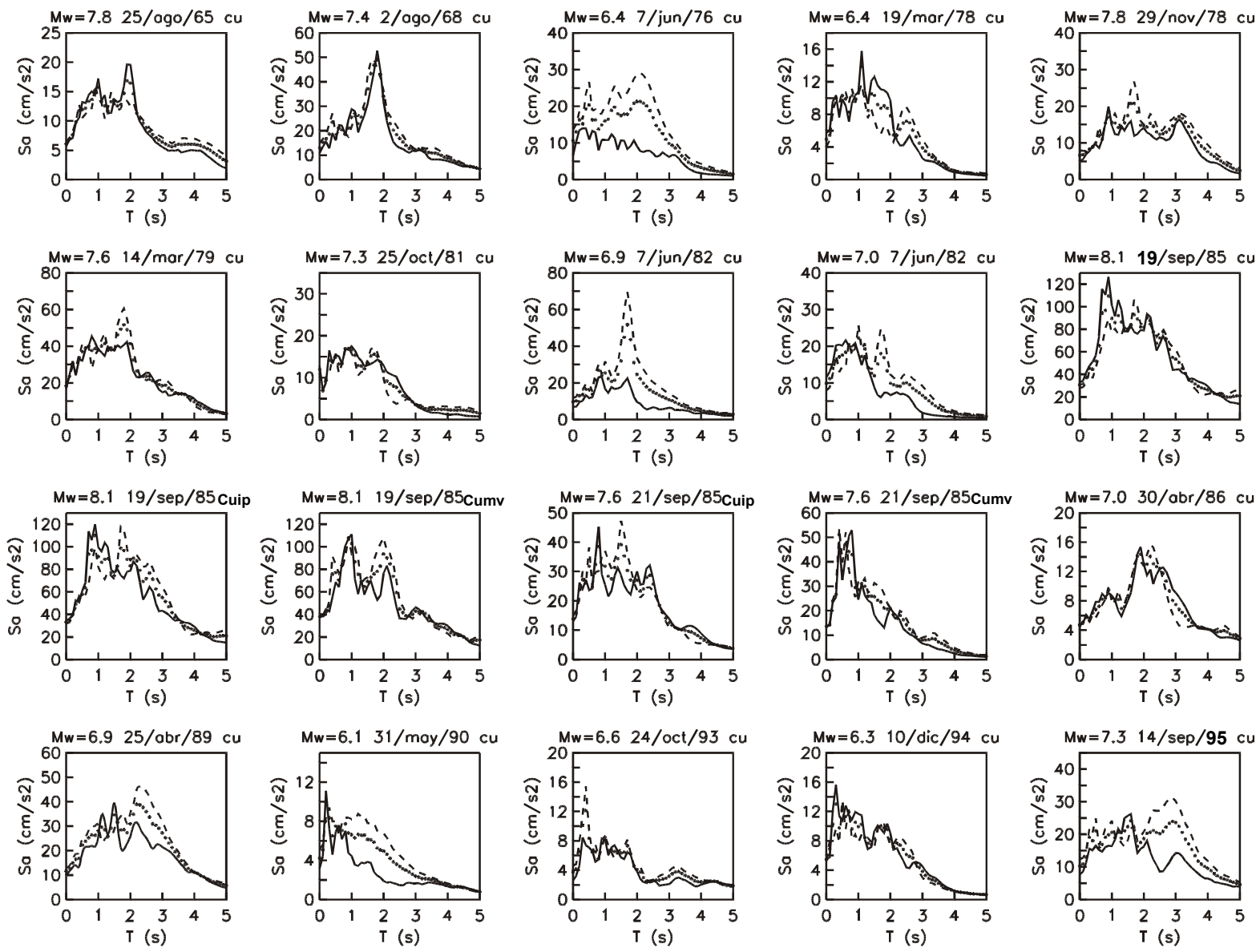

Figura 3. Espectros para 5\% de amortiguamiento registrados en la estación CU de1 23 de agosto de 1965 al 14 de septiembre de 1995.

\section{Cálculo de la forma espectral normalizada}

Haciendo uso del procedimiento mencionado en la introducción y de los datos de la sección anterior se calculó una forma espectral normalizada promedio para los componentes EW, NS, y la media geométrica de la aceleración espectral de ambos componentes del movimiento. Como ejemplo, en la fig. 4 se muestra la forma espectral normalizada promedio (línea de mayor espesor). En la misma figura, aparecen las formas espectrales normalizadas para cada uno de los sismos considerados en este estudio; en ellas se observa que la forma espectral normalizada no es constante de un sismo a otro y presenta una dispersión considerable. Como una medida de la dispersión, en la fig. 5 se muestra la desviación estándar y coeficiente de variación del logaritmo natural de las formas espectrales normalizadas. En ella se observa que ambos parámetros crecen con el periodo. 


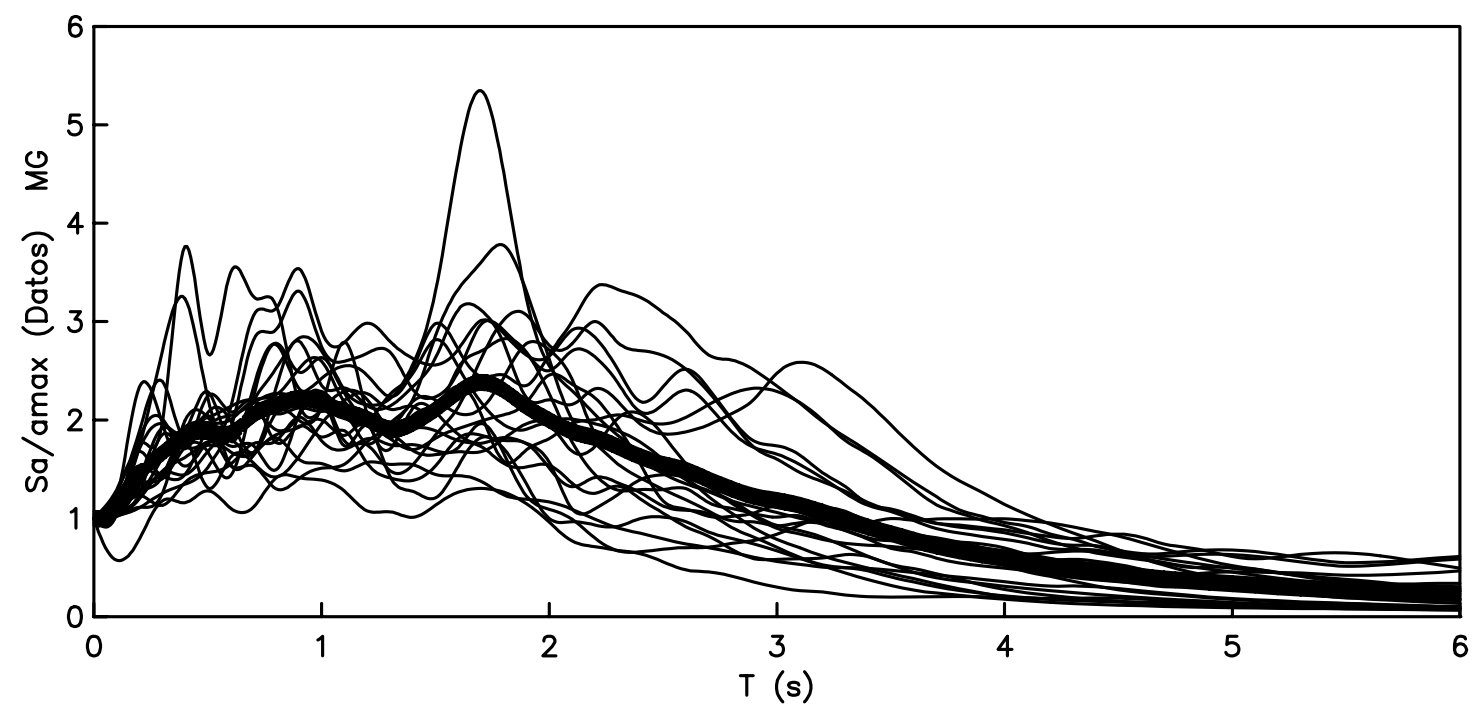

Figura 4. Forma espectral normalizada correspondiente a la media geométrica de la aceleración espectral de los dos componentes horizontales del movimiento del terreno para la estación CU.

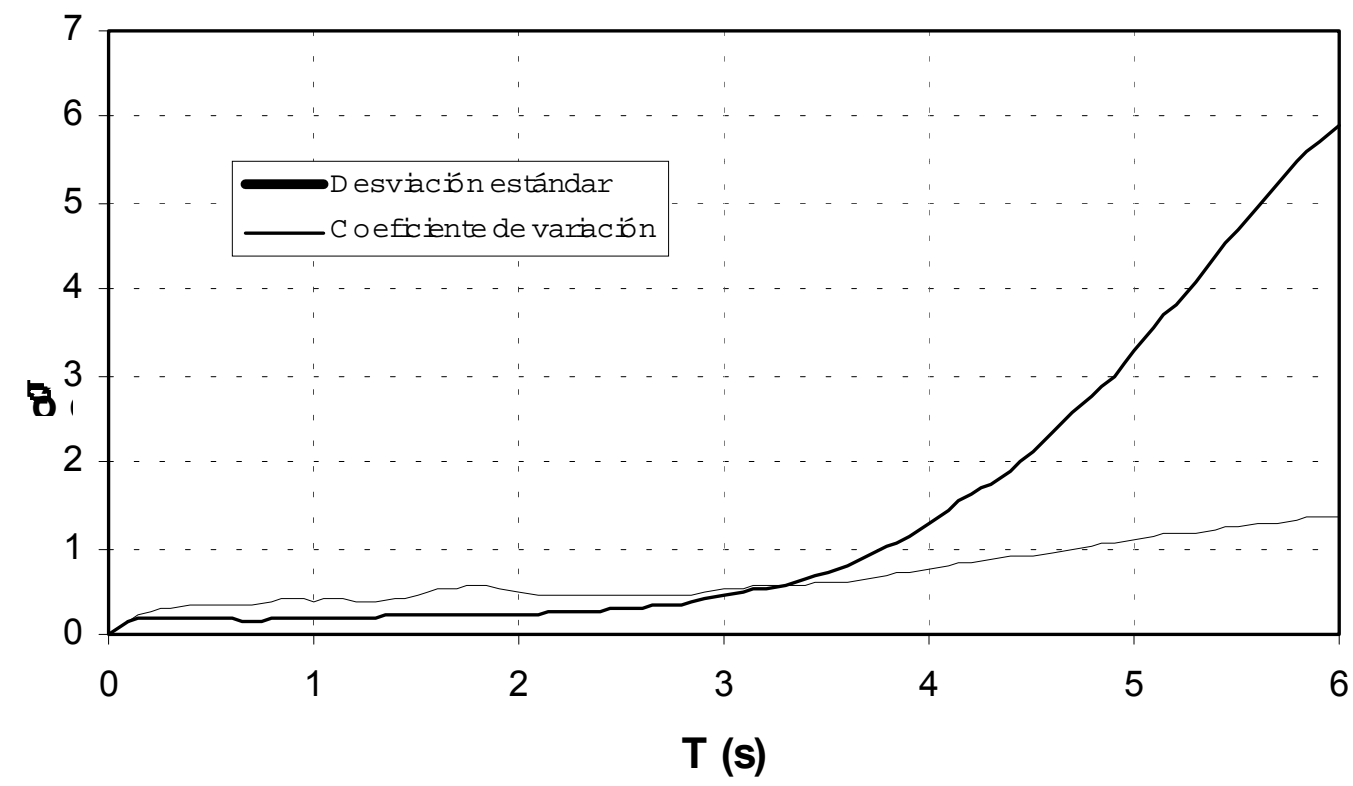

Figura 5. Desviación estándar y coeficiente de variación del logaritmo natural de las formas espectrales normalizadas correspondientes a la media geométrica de la aceleración espectral.

Es importante destacar que la forma del espectro normalizado de los movimientos registrados en la estación $\mathrm{CU}$, para las distancias usadas en este estudio, difiere considerablemente de la que se obtiene de movimientos registrados cercanos al epicentro. Asimismo, en la forma espectral normalizada promedio se aprecia que existe intensidad importante del movimiento aún para periodos cercanos a dos segundos. Ésta, es una posible explicación de las grandes intensidades de movimiento que se presentan en la zona de suelo blando del valle. 


\section{Cálculo de la ley de atenuación espectral}

\section{Funcional para la ley de atenuación espectral}

Para la ley de atenuación espectral se usó el mismo funcional propuesto por Joyner y Boore (1988), el cual está dado por:

$\operatorname{Ln} S_{a}(T)=\alpha_{1}(T)+\alpha_{2}(T)\left(M_{w}-6\right)+\alpha_{3}(T)\left(M_{w}-6\right)^{2}+\alpha_{4}(T) \operatorname{LnR}+\alpha_{5}(T) R+\varepsilon(T)$

donde:

$S a(T)=$ es la aceleración espectral en $\mathrm{cm} / \mathrm{s}^{2}$;

$T=$ es el periodo del sistema de un grado de libertad en $\mathrm{s}$;

$R=$ es la distancia más corta del sitio al área de ruptura en $\mathrm{km}$;

$\alpha_{\mathrm{i}}(T)=$ son los coeficientes a ser calculados a través de una regresión;

$\varepsilon(T)=$ es el error que se comete al estimar el espectro de respuesta con la ley de atenuación espectral.

\section{Regresión para obtener los coeficientes $\alpha_{i}(T)$}

Se siguió un modelo de regresión lineal bayesiana, inspirado en el trabajo de Ordaz, Singh y Arciniega (1994). Un esquema de este tipo de regresión supone que $\alpha_{i}(T)$ y $\sigma(T)$ (desviación estándar del error que se comente al estimar el espectro de respuesta con la ley de atenuación espectral) son variables aleatorias. Por tanto, la diferencia entre la regresión lineal bayesiana y una regresión clásica, como mínimos cuadrados, es que se reconocen como variables aleatorias los coeficientes $\alpha_{i}(T)$.

En resumen, el procedimiento de la regresión lineal bayesiana consiste en estimar los valores esperados a priori de las variables aleatorias, para después actualizarlos a través del teorema de Bayes, obteniendo con ello los valores esperados posteriores de $\alpha_{i}(T)$ y $\sigma(T)$. Los valores esperados a priori de estos parámetros se estiman en función al conocimiento previo que se tenga de ellos y sin hacer intervenir los datos registrados. Para tal efecto, en el presente trabajo se hizo uso del modelo sismológico omega cuadrada, de la teoría de vibraciones aleatorias, de las características geológicas del suelo en que se encuentra localizada la estación CU y de datos de dispersión empleados por Joyner y Boore (1988). Los detalles de la regresión se encuentran en Reyes (1999).

\section{Valores esperados posteriores de $\alpha_{i}(T) y \sigma(T)$}

Los valores esperados posteriores $E^{\prime \prime}\left[\alpha_{i}(T)\right]$, se listan en las tablas 1,2 y 3 , y corresponden a los componentes EW, NS y la media geométrica de la aceleración espectral de ambos componentes del movimiento. 
Estimación de Espectros de Aceleraciones Correspondientes a Diferentes Periodos de Retorno para las Distintas ...

\begin{tabular}{|c|c|c|c|c|c|}
\hline \multicolumn{6}{|c|}{ Tabla 1 Valores esperados posteriores de $\alpha_{i}(T)$ para el componente $\mathbf{E W}$} \\
\hline$T(s)$ & $\mathbf{E}^{\prime \prime}\left[\alpha_{1}(\mathbf{T})\right]$ & $\mathbf{E}^{\prime \prime}\left[\alpha_{2}(\mathbf{T})\right]$ & $\mathbf{E}^{\prime \prime}\left[\alpha_{3}(\mathbf{T})\right]$ & $\mathbf{E}^{\prime \prime}\left[\alpha_{4}(\mathbf{T})\right]$ & $\mathbf{E}^{\prime \prime}\left[\alpha_{5}(\mathbf{T})\right]$ \\
\hline 0.0 & 5.3238 & 1.4781 & -0.0023 & -0.5000 & -0.0058 \\
\hline 0.1 & 6.0807 & 1.5004 & 0.0160 & -0.5000 & -0.0077 \\
\hline 0.2 & 7.0068 & 1.3850 & 0.0297 & -0.5000 & -0.0092 \\
\hline 0.3 & 6.5153 & 1.3126 & 0.0085 & -0.5000 & -0.0072 \\
\hline 0.4 & 6.2363 & 1.5644 & -0.0092 & -0.5000 & -0.0070 \\
\hline 0.5 & 6.0533 & 1.4176 & 0.0170 & -0.5000 & -0.0060 \\
\hline 0.6 & 6.3706 & 1.4745 & 0.0287 & -0.5000 & -0.0070 \\
\hline 0.7 & 6.0709 & 1.6084 & 0.0744 & -0.5000 & -0.0066 \\
\hline 0.8 & 5.9267 & 1.6274 & 0.0659 & -0.5000 & -0.0060 \\
\hline 0.9 & 5.7867 & 1.5710 & 0.0581 & -0.5000 & -0.0053 \\
\hline 1.0 & 5.7609 & 1.5490 & 0.0438 & -0.5000 & -0.0052 \\
\hline 1.1 & 6.2278 & 1.6413 & 0.0254 & -0.5000 & -0.0070 \\
\hline 1.2 & 6.0934 & 1.6405 & 0.0294 & -0.5000 & -0.0067 \\
\hline 1.3 & 6.1538 & 1.6819 & 0.0566 & -0.5000 & -0.0073 \\
\hline 1.4 & 6.1974 & 1.7143 & 0.0134 & -0.5000 & -0.0074 \\
\hline 1.5 & 6.1156 & 1.6489 & 0.0099 & -0.5000 & -0.0068 \\
\hline 1.6 & 5.9344 & 1.6355 & 0.0151 & -0.5000 & -0.0062 \\
\hline 1.7 & 5.5105 & 1.6326 & -0.0194 & -0.5000 & -0.0050 \\
\hline 1.8 & 5.0881 & 1.5917 & -0.0434 & -0.5000 & -0.0035 \\
\hline 1.9 & 5.0848 & 1.6673 & -0.0287 & -0.5000 & -0.0038 \\
\hline 2.0 & 5.0326 & 1.7463 & 0.0051 & -0.5000 & -0.0043 \\
\hline 2.1 & 4.9109 & 1.8251 & 0.0327 & -0.5000 & -0.0046 \\
\hline 2.2 & 4.9375 & 1.8176 & 0.0370 & -0.5000 & -0.0050 \\
\hline 2.3 & 4.9622 & 1.8235 & 0.0321 & -0.5000 & -0.0053 \\
\hline 2.4 & 4.7980 & 1.7637 & -0.0102 & -0.5000 & -0.0046 \\
\hline 2.5 & 4.6260 & 1.7211 & -0.0099 & -0.5000 & -0.0041 \\
\hline 2.6 & 4.3274 & 1.6494 & 0.0043 & -0.5000 & -0.0031 \\
\hline 2.7 & 4.3219 & 1.5990 & 0.0190 & -0.5000 & -0.0032 \\
\hline 2.8 & 4.3588 & 1.5542 & 0.0164 & -0.5000 & -0.0033 \\
\hline 2.9 & 4.2913 & 1.5597 & 0.0120 & -0.5000 & -0.0033 \\
\hline 3.0 & 4.1661 & 1.5755 & 0.0245 & -0.5000 & -0.0032 \\
\hline 3.1 & 4.1480 & 1.5565 & 0.0320 & -0.5000 & -0.0032 \\
\hline 3.2 & 4.1066 & 1.5370 & 0.0331 & -0.5000 & -0.0032 \\
\hline 3.3 & 4.0720 & 1.5046 & 0.0371 & -0.5000 & -0.0032 \\
\hline 3.4 & 4.0095 & 1.5034 & 0.0354 & -0.5000 & -0.0032 \\
\hline 3.5 & 3.9010 & 1.5234 & 0.0511 & -0.5000 & -0.0033 \\
\hline 3.6 & 3.7843 & 1.5740 & 0.0510 & -0.5000 & -0.0033 \\
\hline 3.7 & 3.6278 & 1.6207 & 0.0570 & -0.5000 & -0.0033 \\
\hline 3.8 & 3.4649 & 1.6455 & 0.0672 & -0.5000 & -0.0032 \\
\hline 3.9 & 3.3606 & 1.6563 & 0.0662 & -0.5000 & -0.0031 \\
\hline 4.0 & 3.2924 & 1.6536 & 0.0561 & -0.5000 & -0.0030 \\
\hline 4.1 & 3.2046 & 1.6442 & 0.0432 & -0.5000 & -0.0029 \\
\hline 4.2 & 3.1251 & 1.6256 & 0.0341 & -0.5000 & -0.0027 \\
\hline 4.3 & 3.1266 & 1.5747 & 0.0429 & -0.5000 & -0.0028 \\
\hline 4.4 & 3.1046 & 1.5391 & 0.0430 & -0.5000 & -0.0028 \\
\hline 4.5 & 3.0648 & 1.5187 & 0.0361 & -0.5000 & -0.0028 \\
\hline 4.6 & 3.0218 & 1.5151 & 0.0245 & -0.5000 & -0.0028 \\
\hline 4.7 & 2.9787 & 1.5202 & 0.0157 & -0.5000 & -0.0029 \\
\hline 4.8 & 2.9317 & 1.5233 & 0.0137 & -0.5000 & -0.0029 \\
\hline 4.9 & 2.8880 & 1.5276 & 0.0114 & -0.5000 & -0.0030 \\
\hline 5.0 & 2.8487 & 1.5278 & 0.0078 & -0.5000 & -0.0030 \\
\hline 5.1 & 2.8148 & 1.5270 & 0.0052 & -0.5000 & -0.0031 \\
\hline 5.2 & 2.7673 & 1.5376 & -0.0003 & -0.5000 & -0.0031 \\
\hline 5.3 & 2.7161 & 1.5532 & -0.0032 & -0.5000 & -0.0032 \\
\hline 5.4 & 2.6760 & 1.5660 & -0.0017 & -0.5000 & -0.0032 \\
\hline 5.5 & 2.6446 & 1.5728 & 0.0076 & -0.5000 & -0.0033 \\
\hline 5.6 & 2.5928 & 1.5893 & 0.0304 & -0.5000 & -0.0035 \\
\hline 5.7 & 2.5501 & 1.5985 & 0.0526 & -0.5000 & -0.0036 \\
\hline 5.8 & 2.5078 & 1.5953 & 0.0768 & -0.5000 & -0.0037 \\
\hline 5.9 & 2.4672 & 1.5780 & 0.0963 & -0.5000 & -0.0037 \\
\hline 6.0 & 2.4436 & 1.5664 & 0.1096 & -0.5000 & -0.0037 \\
\hline
\end{tabular}


Carlos Reyes, Eduardo Miranda, Mario Ordaz y Roberto Meli

Tabla 2 Valores esperados posteriores de $\alpha_{i}(T)$ para el componente NS

\begin{tabular}{|c|c|c|c|c|c|}
\hline$T(s)$ & $E^{\prime \prime}\left[\alpha_{1}(T)\right]$ & $\mathbf{E}^{\prime \prime}\left[\alpha_{2}(\mathbf{T})\right]$ & $\mathbf{E}^{\prime \prime}\left[\alpha_{3}(\mathbf{T})\right]$ & $\mathbf{E}^{\prime \prime}\left[\alpha_{4}(\mathbf{T})\right]$ & $\mathbf{E}^{\prime \prime}\left[\alpha_{5}(\mathbf{T})\right]$ \\
\hline 0.0 & 5.6403 & 1.3723 & -0.0197 & -0.5000 & -0.0058 \\
\hline 0.1 & 6.1286 & 1.3875 & -0.0118 & -0.5000 & -0.0070 \\
\hline 0.2 & 6.7888 & 1.3618 & -0.0143 & -0.5000 & -0.0082 \\
\hline 0.3 & 6.8341 & 1.3601 & 0.0031 & -0.5000 & -0.0080 \\
\hline 0.4 & 6.7467 & 1.4099 & 0.0149 & -0.5000 & -0.0077 \\
\hline 0.5 & 7.2087 & 1.4749 & 0.0232 & -0.5000 & -0.0092 \\
\hline 0.6 & 6.5055 & 1.4913 & 0.0356 & -0.5000 & -0.0075 \\
\hline 0.7 & 6.5817 & 1.4479 & 0.0173 & -0.5000 & -0.0072 \\
\hline 0.8 & 6.1242 & 1.4028 & 0.0356 & -0.5000 & -0.0057 \\
\hline 0.9 & 5.9220 & 1.4046 & 0.0452 & -0.5000 & -0.0050 \\
\hline 1.0 & 6.3045 & 1.4549 & 0.0157 & -0.5000 & -0.0061 \\
\hline 1.1 & 6.2865 & 1.4315 & 0.0397 & -0.5000 & -0.0062 \\
\hline 1.2 & 6.4468 & 1.4395 & 0.0374 & -0.5000 & -0.0068 \\
\hline 1.3 & 6.2425 & 1.4168 & 0.0365 & -0.5000 & -0.0063 \\
\hline 1.4 & 6.0595 & 1.4012 & 0.0353 & -0.5000 & -0.0055 \\
\hline 1.5 & 5.9610 & 1.5011 & -0.0085 & -0.5000 & -0.0052 \\
\hline 1.6 & 6.0313 & 1.5732 & -0.0378 & -0.5000 & -0.0052 \\
\hline 1.7 & 5.9630 & 1.5669 & -0.0241 & -0.5000 & -0.0048 \\
\hline 1.8 & 5.5076 & 1.4687 & -0.0261 & -0.5000 & -0.0032 \\
\hline 1.9 & 5.4364 & 1.4389 & -0.0119 & -0.5000 & -0.0034 \\
\hline 2.0 & 5.3720 & 1.4116 & 0.0103 & -0.5000 & -0.0035 \\
\hline 2.1 & 5.3083 & 1.3611 & 0.0213 & -0.5000 & -0.0033 \\
\hline 2.2 & 5.3287 & 1.3508 & 0.0194 & -0.5000 & -0.0034 \\
\hline 2.3 & 5.3475 & 1.3625 & 0.0139 & -0.5000 & -0.0036 \\
\hline 2.4 & 5.3630 & 1.3605 & 0.0036 & -0.5000 & -0.0037 \\
\hline 2.5 & 5.3404 & 1.3573 & -0.0054 & -0.5000 & -0.0038 \\
\hline 2.6 & 5.2978 & 1.3624 & 0.0003 & -0.5000 & -0.0038 \\
\hline 2.7 & 5.2084 & 1.3722 & -0.0067 & -0.5000 & -0.0037 \\
\hline 2.8 & 5.0890 & 1.3947 & -0.0080 & -0.5000 & -0.0037 \\
\hline 2.9 & 4.9654 & 1.4197 & -0.0031 & -0.5000 & -0.0037 \\
\hline 3.0 & 4.8588 & 1.4454 & 0.0076 & -0.5000 & -0.0037 \\
\hline 3.1 & 4.7738 & 1.4901 & 0.0037 & -0.5000 & -0.0037 \\
\hline 3.2 & 4.6637 & 1.5212 & -0.0004 & -0.5000 & -0.0036 \\
\hline 3.3 & 4.5662 & 1.5426 & -0.0070 & -0.5000 & -0.0036 \\
\hline 3.4 & 4.4160 & 1.5593 & -0.0180 & -0.5000 & -0.0034 \\
\hline 3.5 & 4.2465 & 1.5778 & -0.0303 & -0.5000 & -0.0032 \\
\hline 3.6 & 4.1263 & 1.5867 & -0.0466 & -0.5000 & -0.0030 \\
\hline 3.7 & 3.9851 & 1.5983 & -0.0553 & -0.5000 & -0.0028 \\
\hline 3.8 & 3.8512 & 1.6104 & -0.0627 & -0.5000 & -0.0027 \\
\hline 3.9 & 3.7136 & 1.6131 & -0.0607 & -0.5000 & -0.0025 \\
\hline 4.0 & 3.5766 & 1.6188 & -0.0533 & -0.5000 & -0.0024 \\
\hline 4.1 & 3.4876 & 1.6163 & -0.0483 & -0.5000 & -0.0024 \\
\hline 4.2 & 3.3819 & 1.6159 & -0.0452 & -0.5000 & -0.0023 \\
\hline 4.3 & 3.2840 & 1.6116 & -0.0373 & -0.5000 & -0.0022 \\
\hline 4.4 & 3.1909 & 1.6151 & -0.0319 & -0.5000 & -0.0021 \\
\hline 4.5 & 3.1217 & 1.6204 & -0.0364 & -0.5000 & -0.0020 \\
\hline 4.6 & 3.0661 & 1.6246 & -0.0336 & -0.5000 & -0.0021 \\
\hline 4.7 & 3.0276 & 1.6315 & -0.0259 & -0.5000 & -0.0022 \\
\hline 4.8 & 2.9816 & 1.6388 & -0.0121 & -0.5000 & -0.0023 \\
\hline 4.9 & 2.9517 & 1.6355 & 0.0022 & -0.5000 & -0.0024 \\
\hline 5.0 & 2.9062 & 1.6372 & 0.0127 & -0.5000 & -0.0025 \\
\hline 5.1 & 2.8515 & 1.6301 & 0.0205 & -0.5000 & -0.0025 \\
\hline 5.2 & 2.7857 & 1.6253 & 0.0236 & -0.5000 & -0.0024 \\
\hline 5.3 & 2.7080 & 1.6324 & 0.0217 & -0.5000 & -0.0024 \\
\hline 5.4 & 2.6281 & 1.6276 & 0.0235 & -0.5000 & -0.0023 \\
\hline 5.5 & 2.5427 & 1.6182 & 0.0302 & -0.5000 & -0.0022 \\
\hline 5.6 & 2.4649 & 1.6162 & 0.0352 & -0.5000 & -0.0021 \\
\hline 5.7 & 2.4039 & 1.6169 & 0.0408 & -0.5000 & -0.0021 \\
\hline 5.8 & 2.3562 & 1.6124 & 0.0504 & -0.5000 & -0.0021 \\
\hline 5.9 & 2.3404 & 1.6124 & 0.0593 & -0.5000 & -0.0022 \\
\hline 6.0 & 2.3297 & 1.6199 & 0.0670 & -0.5000 & -0.0024 \\
\hline
\end{tabular}


Estimación de Espectros de Aceleraciones Correspondientes a Diferentes Periodos de Retorno para las Distintas ...

Tabla 3 Valores esperados posteriores de $\alpha_{i}(T)$ para la media geométrica

\begin{tabular}{|c|c|c|c|c|c|}
\hline T (s) & $\mathbf{E}^{\prime \prime}\left[\alpha_{1}(\mathbf{T})\right]$ & $\mathbf{E}^{\prime \prime}\left[\alpha_{2}(\mathbf{T})\right]$ & $\mathbf{E}^{\prime \prime}\left[\alpha_{3}(\mathbf{T})\right]$ & $\mathbf{E}^{\prime \prime}\left[\alpha_{4}(\mathbf{T})\right]$ & $\mathbf{E}^{\prime \prime}\left[\alpha_{5}(\mathbf{T})\right]$ \\
\hline 0.0 & 5.5474 & 1.4024 & -0.0130 & -0.5000 & -0.0058 \\
\hline 0.1 & 6.1209 & 1.4348 & -0.0013 & -0.5000 & -0.0073 \\
\hline 0.2 & 6.9358 & 1.3645 & 0.0057 & -0.5000 & -0.0087 \\
\hline 0.3 & 6.7167 & 1.3429 & 0.0018 & -0.5000 & -0.0077 \\
\hline 0.4 & 6.5763 & 1.4632 & 0.0056 & -0.5000 & -0.0074 \\
\hline 0.5 & 6.7032 & 1.4401 & 0.0176 & -0.5000 & -0.0077 \\
\hline 0.6 & 6.4179 & 1.4790 & 0.0307 & -0.5000 & -0.0071 \\
\hline 0.7 & 6.3641 & 1.5249 & 0.0517 & -0.5000 & -0.0070 \\
\hline 0.8 & 6.1296 & 1.5230 & 0.0527 & -0.5000 & -0.0061 \\
\hline 0.9 & 5.9193 & 1.4742 & 0.0554 & -0.5000 & -0.0053 \\
\hline 1.0 & 6.0979 & 1.4830 & 0.0301 & -0.5000 & -0.0057 \\
\hline 1.1 & 6.3256 & 1.4953 & 0.0366 & -0.5000 & -0.0066 \\
\hline 1.2 & 6.3147 & 1.4933 & 0.0427 & -0.5000 & -0.0067 \\
\hline 1.3 & 6.2710 & 1.5000 & 0.0519 & -0.5000 & -0.0068 \\
\hline 1.4 & 6.2372 & 1.5037 & 0.0286 & -0.5000 & -0.0065 \\
\hline 1.5 & 6.1455 & 1.5478 & -0.0021 & -0.5000 & -0.0061 \\
\hline 1.6 & 6.0656 & 1.5745 & -0.0227 & -0.5000 & -0.0056 \\
\hline 1.7 & 5.8514 & 1.5423 & -0.0275 & -0.5000 & -0.0047 \\
\hline 1.8 & 5.5971 & 1.5066 & -0.0293 & -0.5000 & -0.0040 \\
\hline 1.9 & 5.5686 & 1.5109 & -0.0121 & -0.5000 & -0.0042 \\
\hline 2.0 & 5.5895 & 1.5188 & 0.0184 & -0.5000 & -0.0046 \\
\hline 2.1 & 5.4553 & 1.5193 & 0.0341 & -0.5000 & -0.0046 \\
\hline 2.2 & 5.5002 & 1.5379 & 0.0303 & -0.5000 & -0.0049 \\
\hline 2.3 & 5.6003 & 1.5721 & 0.0220 & -0.5000 & -0.0054 \\
\hline 2.4 & 5.6556 & 1.5693 & 0.0022 & -0.5000 & -0.0056 \\
\hline 2.5 & 5.6298 & 1.5592 & 0.0009 & -0.5000 & -0.0057 \\
\hline 2.6 & 5.5949 & 1.5584 & 0.0146 & -0.5000 & -0.0058 \\
\hline 2.7 & 5.4798 & 1.5483 & 0.0129 & -0.5000 & -0.0056 \\
\hline 2.8 & 5.3864 & 1.5496 & 0.0093 & -0.5000 & -0.0055 \\
\hline 2.9 & 5.2736 & 1.5669 & 0.0102 & -0.5000 & -0.0055 \\
\hline 3.0 & 5.1313 & 1.5834 & 0.0178 & -0.5000 & -0.0053 \\
\hline 3.1 & 5.0578 & 1.6068 & 0.0166 & -0.5000 & -0.0053 \\
\hline 3.2 & 4.9390 & 1.6159 & 0.0129 & -0.5000 & -0.0052 \\
\hline 3.3 & 4.8502 & 1.6205 & 0.0114 & -0.5000 & -0.0051 \\
\hline 3.4 & 4.6885 & 1.6233 & 0.0046 & -0.5000 & -0.0048 \\
\hline 3.5 & 4.4781 & 1.6328 & 0.0047 & -0.5000 & -0.0045 \\
\hline 3.6 & 4.3109 & 1.6511 & -0.0022 & -0.5000 & -0.0043 \\
\hline 3.7 & 4.0917 & 1.6647 & -0.0059 & -0.5000 & -0.0039 \\
\hline 3.8 & 3.8577 & 1.6667 & -0.0077 & -0.5000 & -0.0034 \\
\hline 3.9 & 3.6392 & 1.6583 & -0.0095 & -0.5000 & -0.0030 \\
\hline 4.0 & 3.4326 & 1.6456 & -0.0136 & -0.5000 & -0.0025 \\
\hline 4.1 & 3.2699 & 1.6238 & -0.0201 & -0.5000 & -0.0021 \\
\hline 4.2 & 3.1102 & 1.6018 & -0.0268 & -0.5000 & -0.0018 \\
\hline 4.3 & 3.0082 & 1.5663 & -0.0209 & -0.5000 & -0.0015 \\
\hline 4.4 & 2.8670 & 1.5361 & -0.0207 & -0.5000 & -0.0012 \\
\hline 4.5 & 2.7522 & 1.5195 & -0.0283 & -0.5000 & -0.0009 \\
\hline 4.6 & 2.7061 & 1.5235 & -0.0309 & -0.5000 & -0.0010 \\
\hline 4.7 & 2.7290 & 1.5430 & -0.0270 & -0.5000 & -0.0013 \\
\hline 4.8 & 2.7307 & 1.5576 & -0.0167 & -0.5000 & -0.0016 \\
\hline 4.9 & 2.7483 & 1.5672 & -0.0048 & -0.5000 & -0.0019 \\
\hline 5.0 & 2.7511 & 1.5758 & 0.0043 & -0.5000 & -0.0021 \\
\hline 5.1 & 2.7192 & 1.5715 & 0.0110 & -0.5000 & -0.0022 \\
\hline 5.2 & 2.6453 & 1.5661 & 0.0130 & -0.5000 & -0.0021 \\
\hline 5.3 & 2.5565 & 1.5694 & 0.0109 & -0.5000 & -0.0020 \\
\hline 5.4 & 2.4587 & 1.5660 & 0.0107 & -0.5000 & -0.0019 \\
\hline 5.5 & 2.3675 & 1.5589 & 0.0155 & -0.5000 & -0.0018 \\
\hline 5.6 & 2.2935 & 1.5654 & 0.0230 & -0.5000 & -0.0017 \\
\hline 5.7 & 2.2675 & 1.5730 & 0.0322 & -0.5000 & -0.0018 \\
\hline 5.8 & 2.2725 & 1.5759 & 0.0464 & -0.5000 & -0.0021 \\
\hline 5.9 & 2.3118 & 1.5801 & 0.0617 & -0.5000 & -0.0024 \\
\hline 6.0 & 2.3612 & 1.5918 & 0.0749 & -0.5000 & -0.0027 \\
\hline
\end{tabular}




\section{RESULTADOS}

Con excepción de la última parte de esta sección, los resultados que se muestran corresponden a la media geométrica de los componentes EW y NS, para los cuales se obtuvieron tendencias similares. Así, en la fig. 6 se muestran los espectros observados y estimados con la forma espectral normalizada y con la ley de atenuación espectral. En el caso de la forma espectral normalizada, se ha empleado como factor de escalamiento el primer punto de la ley de atenuación espectral, el cual corresponde a la estimación de la aceleración máxima del terreno. A primera vista los espectros estimados con ambos procedimientos parecen aceptables, por lo que, para seleccionar el procedimiento con que mejor se estima el espectro de respuesta en la estación CU, se hace un análisis de los errores que se cometen al estimar el espectro respuesta y se estudia cómo afecta el despreciar el efecto de la magnitud en la forma espectral.
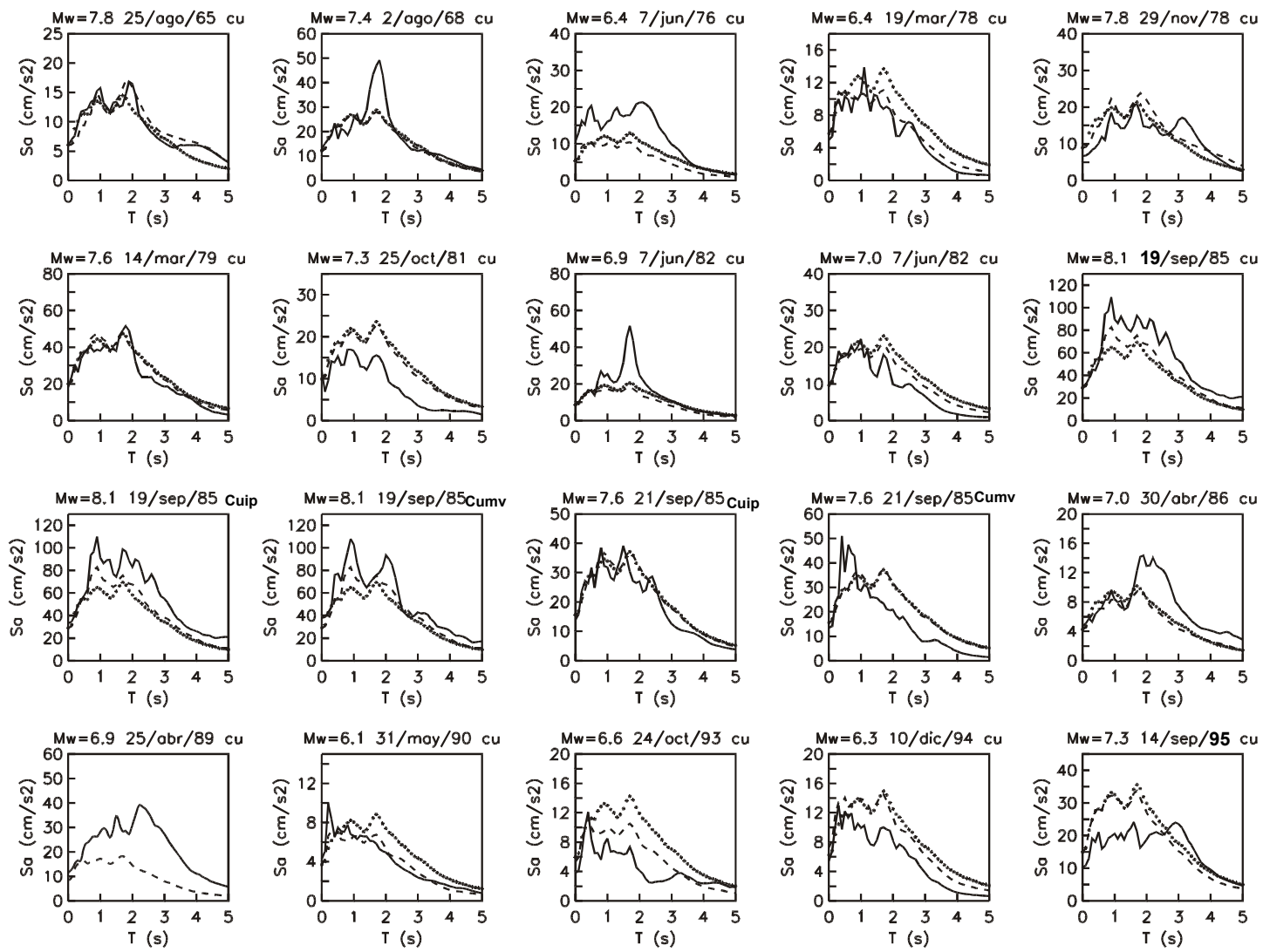

Figura 6. Espectros observados (línea continua) y estimados (con línea punteada cuando se usa la forma espectral normalizada y con línea discontinua cuando se usa la ley de atenuación espectral).

\section{Fuentes de error al estimar el espectro con la forma espectral normalizada}

Las posibles fuentes de error al estimar los espectros con la forma espectral normalizada son:

- La estimación de la aceleración máxima del terreno; 
- Suponer que la forma del espectro no cambia con la magnitud y la distancia.

Con el fin de evaluar la contribución de ambas fuentes de error en la estimación del espectro de respuesta en la estación CU, se construyó la fig. 7. Para el primer caso, en dicha figura se graficó la desviación estándar del error, obteniéndolo de la comparación del espectro observado y el calculado escalando la forma espectral normalizada con la aceleración máxima del terreno estimada con el primer punto de la ley de atenuación espectral. Para el segundo caso, se graficó la desviación estándar del error, calculando éste a partir del espectro observado y el estimado escalando la forma espectral normalizada con la aceleración máxima del terreno observada. La diferencia entre las dos curvas representa la contribución a la incertidumbre relacionada con la estimación de la aceleración máxima del terreno. Tal diferencia es mínima, esto significa que, la mayor incertidumbre se deriva de considerar que la forma espectral no varía con la magnitud y la distancia.

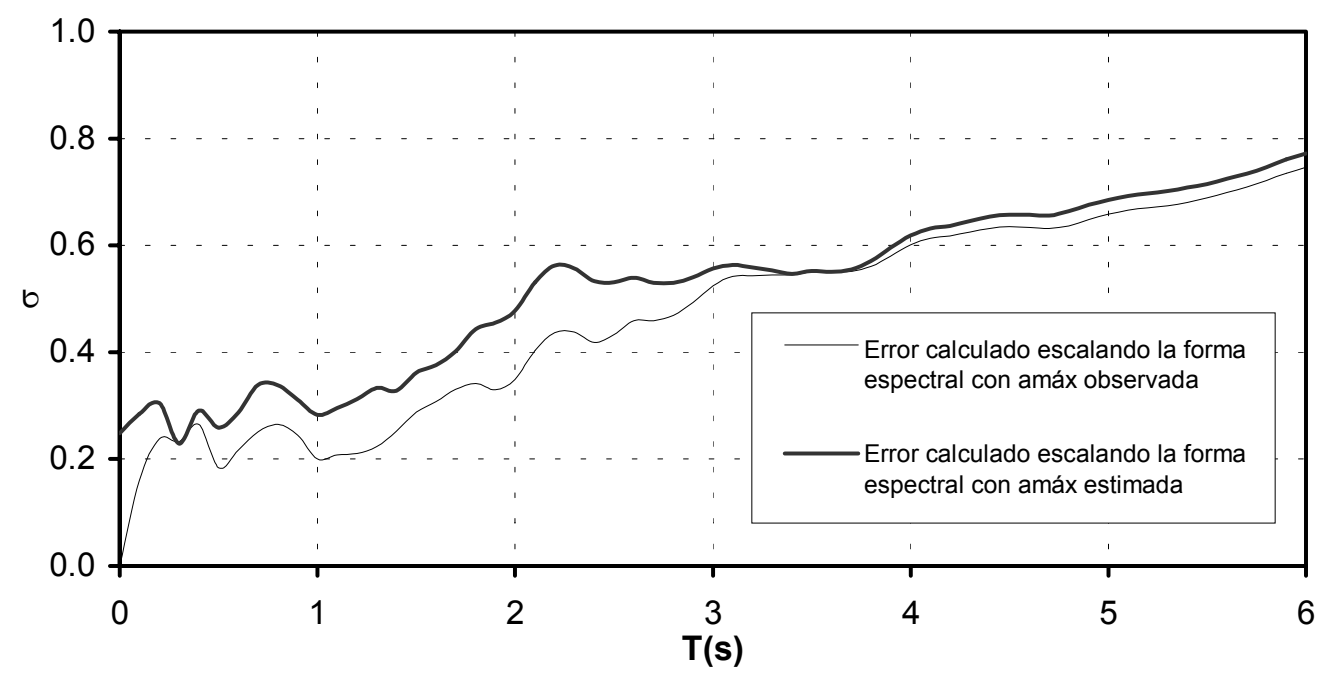

Figura 7. Desviación estándar del error que se comete al estimar el espectro de respuesta en la estación CU con la forma espectral normalizada.

\section{Fuentes de error al estimar el espectro con la ley de atenuación espectral}

En el caso de la ley de atenuación espectral la fuente de error está en el cálculo de los coeficientes $\alpha_{i}(T)$. En la fig. 8 se muestra una comparación de la desviación estándar del error que se comete al estimar el espectro de respuesta con la ley de atenuación espectral, calculada sin considerar que los coeficientes $\alpha_{i}(T)$ son variables aleatorias y la que resulta de aplicar la regresión bayesiana. En esta figura se observa que, al considerar como variables aleatorias los coeficientes $\alpha_{i}(T)$ la desviación estándar del error se incrementa.

\section{Comparación de los errores cometidos con la forma espectral normalizada y con la ley de atenuación espectral}

En la fig. 9 se compara la desviación estándar del error que se comente al estimar el espectro de respuesta con la ley de atenuación espectral y con la forma espectral normalizada. Para poder hacer 
la comparación de ambos procedimientos, se consideró que los coeficientes $\alpha_{i}(T)$ no son variables aleatorias. En la figura se observa que, para todos los periodos se comete mayor error al usar la forma espectral normalizada, lo que representa una ventaja para la ley de atenuación espectral.

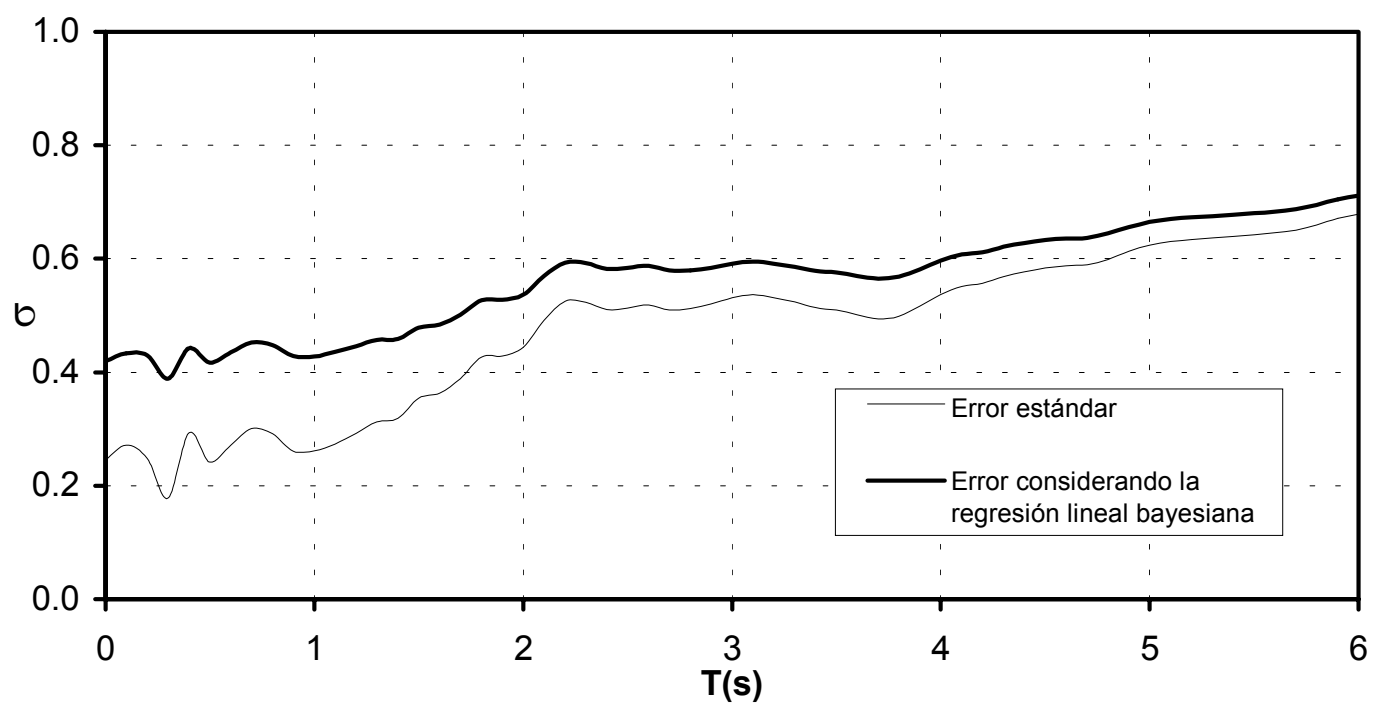

Figura 8. Desviación estándar del error que se comete al estimar el espectro de respuesta en la estación CU considerando y sin considerar que los coeficientes $\alpha_{i}(T)$ son variables aleatorias.

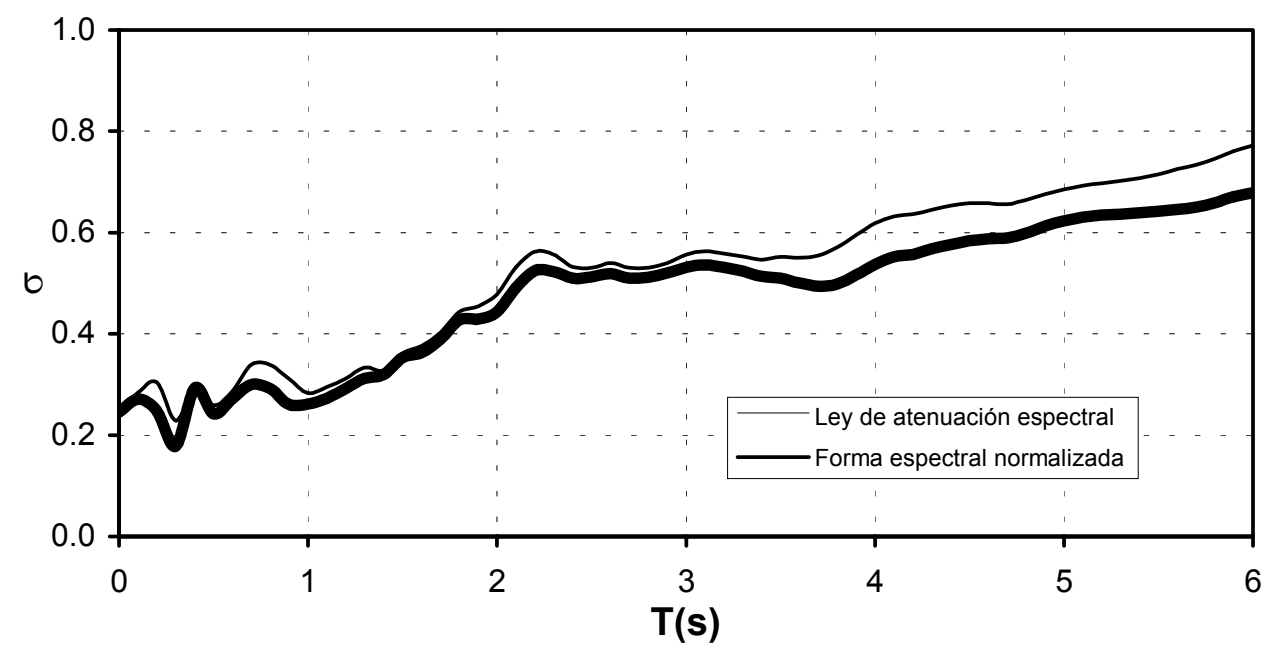

Figura 9. Desviación estándar del error que se comete al estimar el espectro de respuesta en la estación CU con la con la ley de atenuación espectral y la forma espectral normalizada.

\section{Efecto de la magnitud en el espectro de respuesta}

Otro aspecto importante en la estimación del espectro de respuesta es el efecto que sobre éste tiene la magnitud. Dicho efecto se manifiesta de la siguiente manera: a medida que la magnitud del sismo 
crece, la intensidad espectral se amplifica a periodos largos, mientras que para sismos con magnitudes bajas la intensidad espectral se amplifica a periodos cortos. En la estación CU el efecto de la magnitud no se puede observar con claridad, y parecería que tal efecto no tiene influencia en la forma del espectro de respuesta. Por tal razón, en las gráficas de las figs. 10a y 10b se construyó, para la estación 56 y los sismos del 31 de mayo de 1990 y el 14 de septiembre de 1995, el espectro de respuesta observado y el estimado con el uso de los CER y considerando en la estación CU, el espectro estimado con la ley de atenuación espectral y con la forma espectral normalizada; los CER se calcularon de acuerdo al procedimiento de la siguiente sección. La estación 56 está localizada en la zona del lago, donde la mayoría de los espectros de respuesta se caracterizan por tener dos picos.

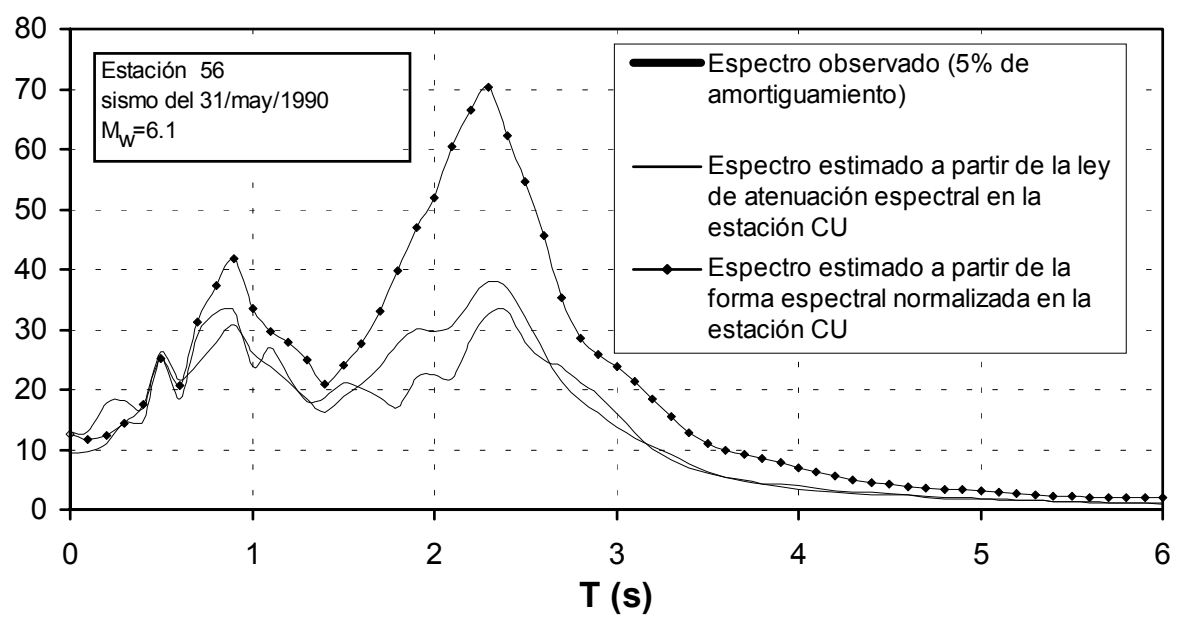

Figura 10a. Efecto de la magnitud en el espectro de respuesta en la estación No. 56 para el sismo del 31 de mayo de 1990, considerando el componente EW del movimiento del terreno.

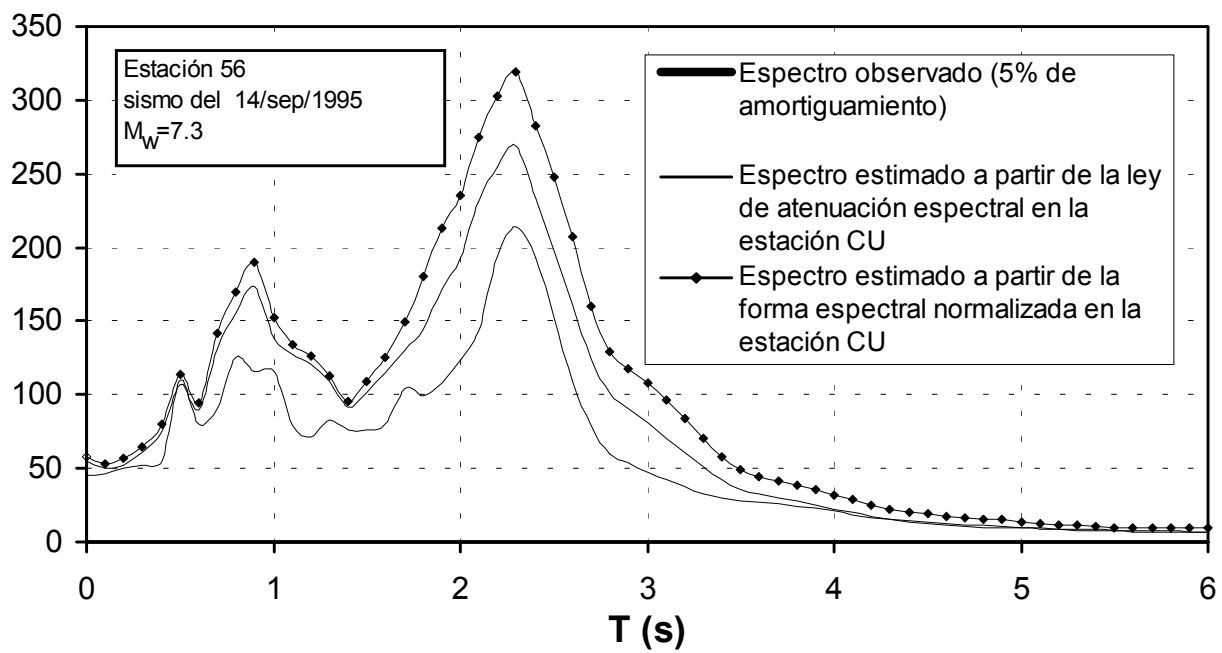

Figura 10b. Efecto de la magnitud en el espectro de respuesta en la estación 56 para el sismo del 14 de septiembre de 1995, considerando el componente EW del movimiento del terreno.

Así, en la fig. 10a se aprecia que para el sismo de mayo de 1990 con magnitud $\mathrm{M}_{\mathrm{w}}=6.1$, en el espectro observado la intensidad de las ordenadas en el primer pico fue prácticamente igual a la del 
segundo, guardando una relación de 0.99; en el espectro estimado con los CER y la ley de atenuación espectral para obtener el espectro en la estación CU, esa relación fue de 1.23. Mientras que, en el espectro estimado con los CER y la forma espectral normalizada para obtener el espectro en la estación CU dicha relación fue 1.67. En el sismo del 14 de septiembre de 1995 con magnitud $\mathrm{M}_{\mathrm{w}}=7.3$, fig. 10b, la intensidad espectral del segundo pico fue superior a la del primero, guardando una relación en el espectro observado de 1.84, para el espectro estimado con los CER y la ley de atenuación espectral para obtener el espectro en la estación CU la relación fue 1.54 y en el espectro estimado con los CER y la forma espectral normalizada para obtener el espectro en la estación CU tal relación se mantuvo constante en 1.67. Al mantenerse constante la relación de la intensidad espectral de los dos picos característicos del espectro en la estación en cuestión, cuando éste se estima con los CER y la forma espectral normalizada para obtener el espectro en la estación CU, se muestra que esta técnica desprecia el efecto de magnitud.

En conclusión, dado que con la ley de atenuación espectral se comete un menor error al estimar el espectro de respuesta en la estación CU y además se tienen en cuenta los efectos de magnitud y distancia, ésta es la técnica que se emplea en lo que resta de este trabajo.

\section{ESTIMACIÓN DEL ESPECTRO DE RESPUESTA EN LAS ZONAS DE TRANSICIÓN Y DEL LAGO}

Con el fin de estimar el espectro de respuesta en las zonas de transición y del lago se usaron los CER y el procedimiento que se describe en la introducción. En este trabajo, los CER se calcularon para un $5 \%$ de amortiguamiento, sin embargo, se reconoce que varían según el nivel del amortiguamiento que se considere. El cálculo de los CER se llevó a cabo como se explica en seguida.

\section{Cálculo de los CER}

El procedimiento que se siguió fue con base en Arciniega et al. (1993) que consiste en calcular, para cada temblor, un espectro promedio para las estaciones de la zona firme $y$, con éste, calcular el CER en las estaciones de la zona de transición o del lago. Una vez conocidos los CER para cada temblor se toma un promedio de todos ellos, el cual se usa para estimar el espectro de respuesta en las zonas de transición y del lago.

En el cálculo de los CER se usaron las estaciones del Instituto de Ingeniería (I de I) y del Centro de Instrumentación y Registro Sísmico (CIRES); los registros obtenidos corresponden a sismos de subducción ocurridos entre el 19 de septiembre de 1985 y el 14 de septiembre de 1995. En la zona firme se consideraron todas las estaciones que administran ambas instituciones; en las zonas de transición y del lago se seleccionaron las estaciones en función del número de sismos que han registrado desde su instalación, la posición que ocupan en la distribución dentro de la ciudad, el periodo dominante del suelo y la amplificación de movimiento que presentan.

Las figs. 11a y $11 \mathrm{~b}$ contienen los CER promedio para la media geométrica de la aceleración espectral de los dos componentes del movimiento horizontal del terreno. Las estaciones 24 y 27 
corresponden a la zona de transición y las restantes a la zona del lago. En los CER mostrados se observa la gran variación de la amplificación del movimiento en la zona de terreno blando de la ciudad de México.
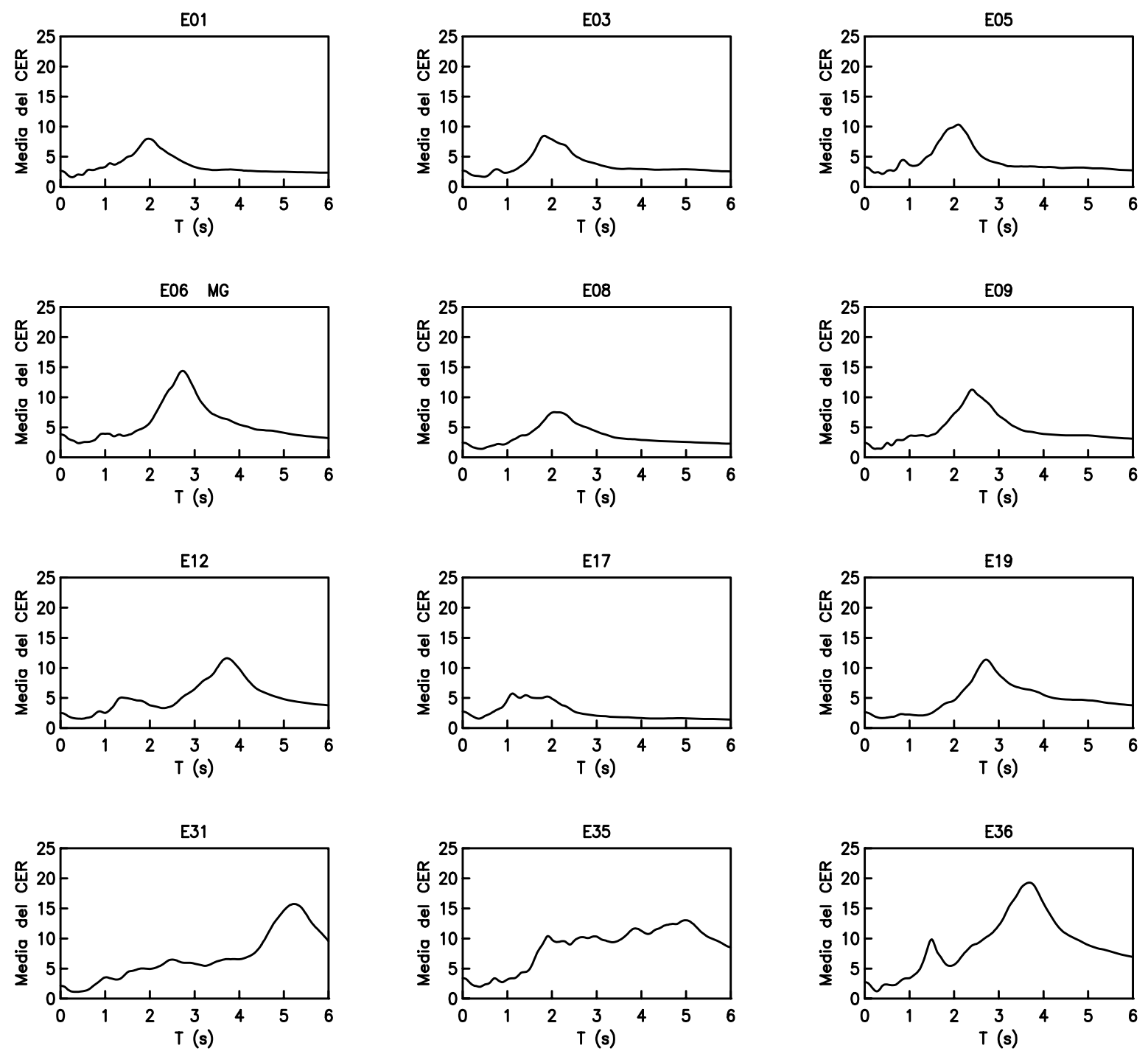

Figura 11a. Cocientes espectrales de respuesta promedio para la media geométrica de la aceleración espectral de los dos componentes del movimiento horizontal del terreno.

\section{Incertidumbre asociada a los CER}

Con el fin de evaluar el efecto de la incertidumbre en los CER al estimar el espectro de respuesta en las zonas de terreno blando se construyeron las figs. 12a y 12b correspondientes a la estaciones SCT (localizada en la Secretaría de Comunicaciones y Transportes en la zona III) y 56. De las dos posibles fuentes de incertidumbre al estimar el espectro en suelo blando, estimación del espectro en terreno firme e incertidumbre en los CER, en las figuras de la izquierda se ha eliminado la primera al 
considerar el espectro observado en la estación CU (método 1), por lo que la diferencia entre el espectro estimado y el observado se debe únicamente al CER. Estas figuras muestran que el uso de los CER para estimar espectros de respuesta en suelo blando conduce a resultados aceptables. Mientras que de las figuras de la derecha puede verse que al reemplazar el espectro observado en terreno firme por el estimado con la ley de atenuación espectral (método 2), se incrementa el error en la estimación del espectro en terreno blando. Ello implica, en términos generales, que la estimación del espectro en suelo blando con los CER gana precisión en la medida que se mejore el espectro estimado en la zona de terreno firme. El estudio de Arciniega et al. (1993) también muestra que, el uso de los CER para estimar espectros en diferentes sitios de la zona de terreno blando conduce a resultados satisfactorios.
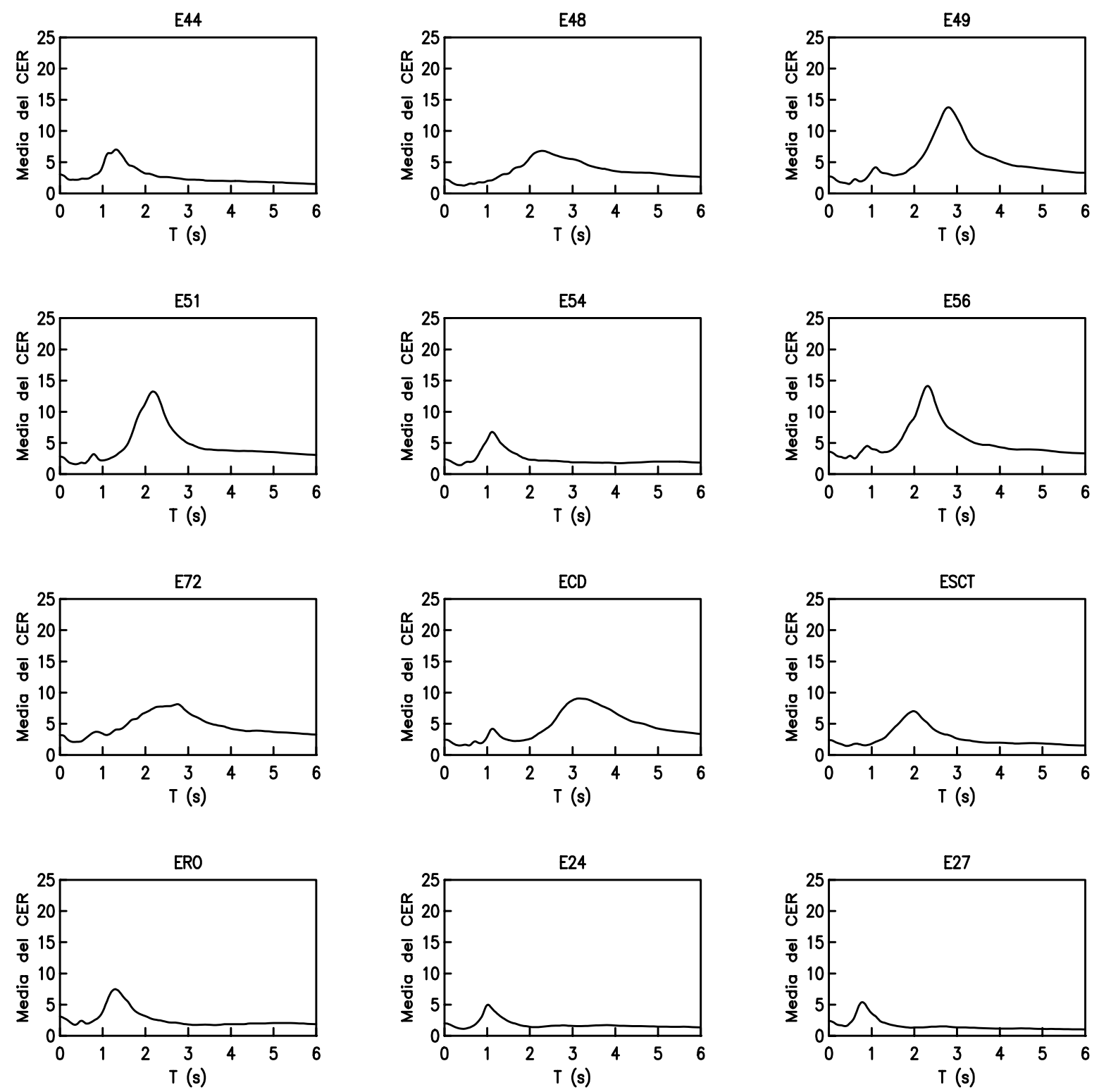

Figura 11b. Cocientes espectrales de respuesta promedio para la media geométrica de la aceleración espectral de los dos componentes del movimiento horizontal del terreno. 

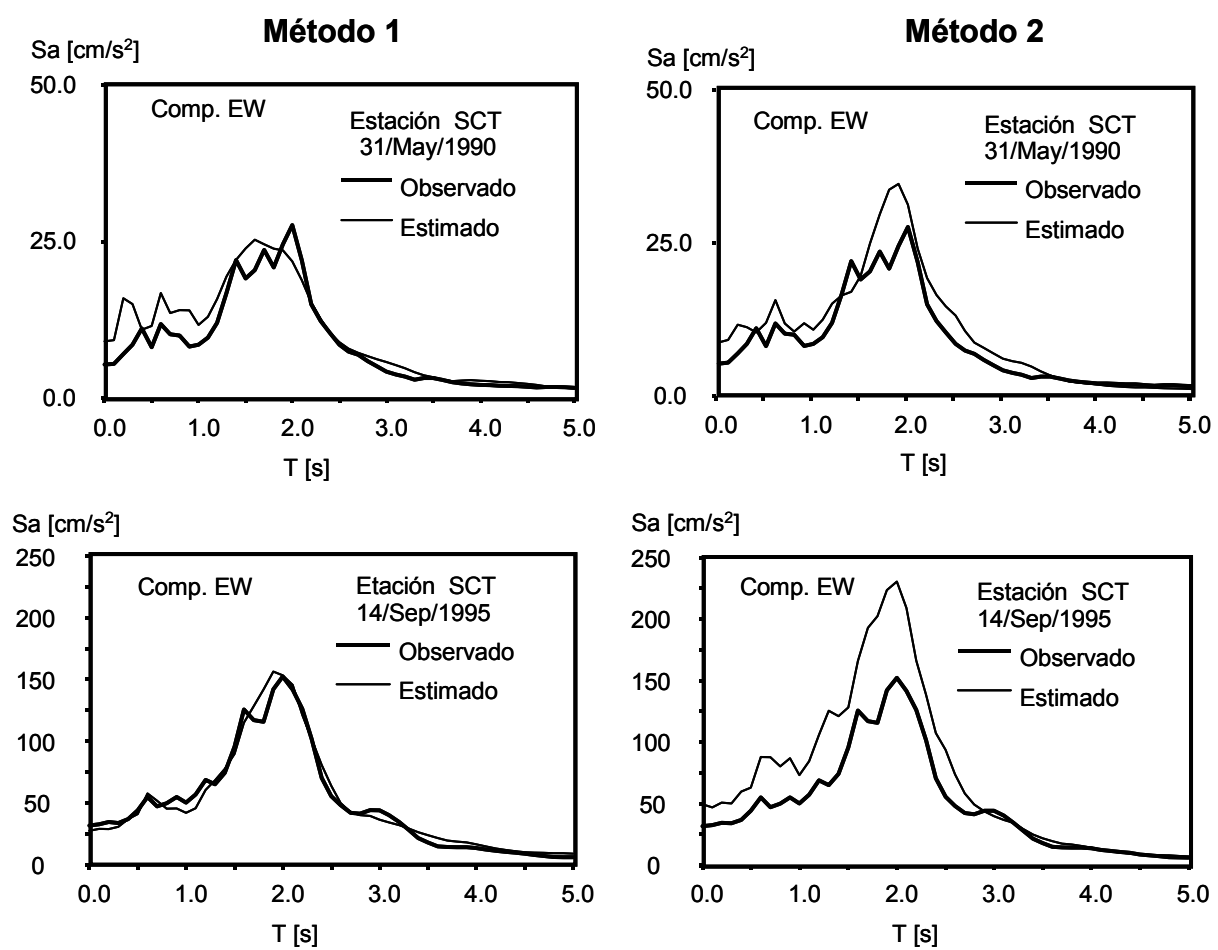

Figura 12a. Espectros de 5\% de amortiguamiento observados vs. estimados con los cocientes espectrales para el componente EW en la estación SCT.
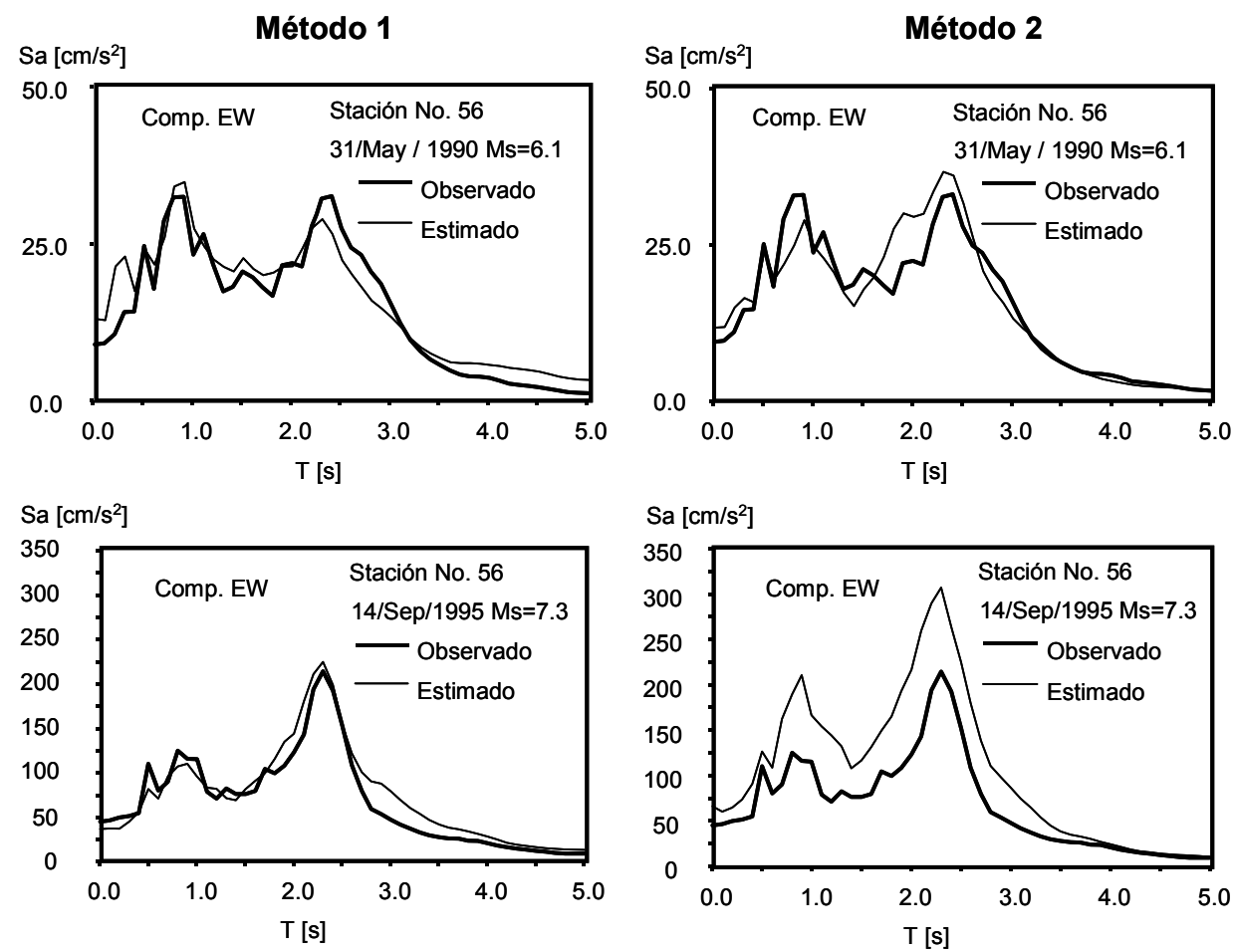

Figura 12b. Espectros de 5\% de amortiguamiento observados vs. estimados con los cocientes espectrales para el componente EW en la estación 56. 


\section{CÁlCULO DE LAS CURVAS DE PELIGRO SÍSMICO Y ESTIMACIÓN DE ESPECTROS DE RESPUESTA ASOCIADOS A DISTINTOS PERIODOS DE RETORNO}

Esta sección describe, de forma general, el procedimiento que permite el cálculo de las curvas de peligro sísmico, mediante las cuales se estima el espectro de respuesta con distintos períodos de retorno en los diferentes tipos de suelo de la ciudad de México.

\section{Catálogo de Sismos}

El catálogo de sismos con que se contó en el estudio fue el de Zúñiga (1996), del cual sólo se consideraron sismos superficiales ocurridos en la costa del Pacífico mexicano registrados de 1900 a 1995 con magnitudes $\mathrm{M}_{\mathrm{s}}$ a partir de 4.5. El contenido de este catálogo, se corroboró con el catálogo publicado por Singh et al.(1984). Aunque el catálogo de Zúñiga (1996) es para magnitud $\mathrm{M}_{\mathrm{s}}$, ésta es aproximadamente igual en valor a $\mathrm{M}_{\mathrm{w}}$ en el intervalo de magnitudes que se manejó en la ley de atenuación espectral que se propone en este trabajo.

Del análisis del catálogo resultó que está completo para magnitudes de 7.0 ó mayores de 1900 a 1995, para magnitudes de 6.0 ó mayores de 1955 a 1995, y para magnitudes de 4.5 ó mayores de 1965 a 1995.

\section{Curvas de excedencia y espectros de peligro uniforme}

Las curvas de excedencia se calcularon únicamente para la estación CU. En el caso de las estaciones localizadas en suelo blando, las curvas de peligro sísmico se construyeron con base en las curvas de la estación CU y los CER, esto es, una vez calculadas las curvas de peligro sísmico en la estación $\mathrm{CU}$ se multiplican por el CER correspondiente y se obtuvieron las curvas de peligro sísmico en las estaciones de la zona de transición o del lago; implícitamente, con este procedimiento se desprecia la incertidumbre asociada con los CER.

En el cálculo de las curvas de excedencia para la estación CU, se tomó como base la ley de atenuación espectral que se desarrolla en este trabajo. Asimismo, se consideraron dos grupos de sismos y fuentes sísmicas asociadas a ellos. El primer grupo, toma en cuenta los sismos pequeños a moderados con magnitud menor a 7; en este caso, se considera que son 4 las fuentes sísmicas de la costa del Pacífico que pueden generar sismos con las características anteriores. En un segundo grupo se consideraron los sismos característicos con magnitud mayor a 7; este tipo de sismos son generados por 14 fuentes sísmicas. Los detalles de las fuentes sísmicas, sus parámetros y las ecuaciones que definen las curvas de excedencia se pueden consultar en Ordaz y Reyes (1999).

En la estación CU resultaron curvas de excedencia como las mostradas en las gráficas de la fig. 13. En la misma figura se comparan estas curvas con las obtenidas de los espectros de respuesta producto de los registros acelerográficos en la estación CU. De dicha comparación se puede comentar que: 

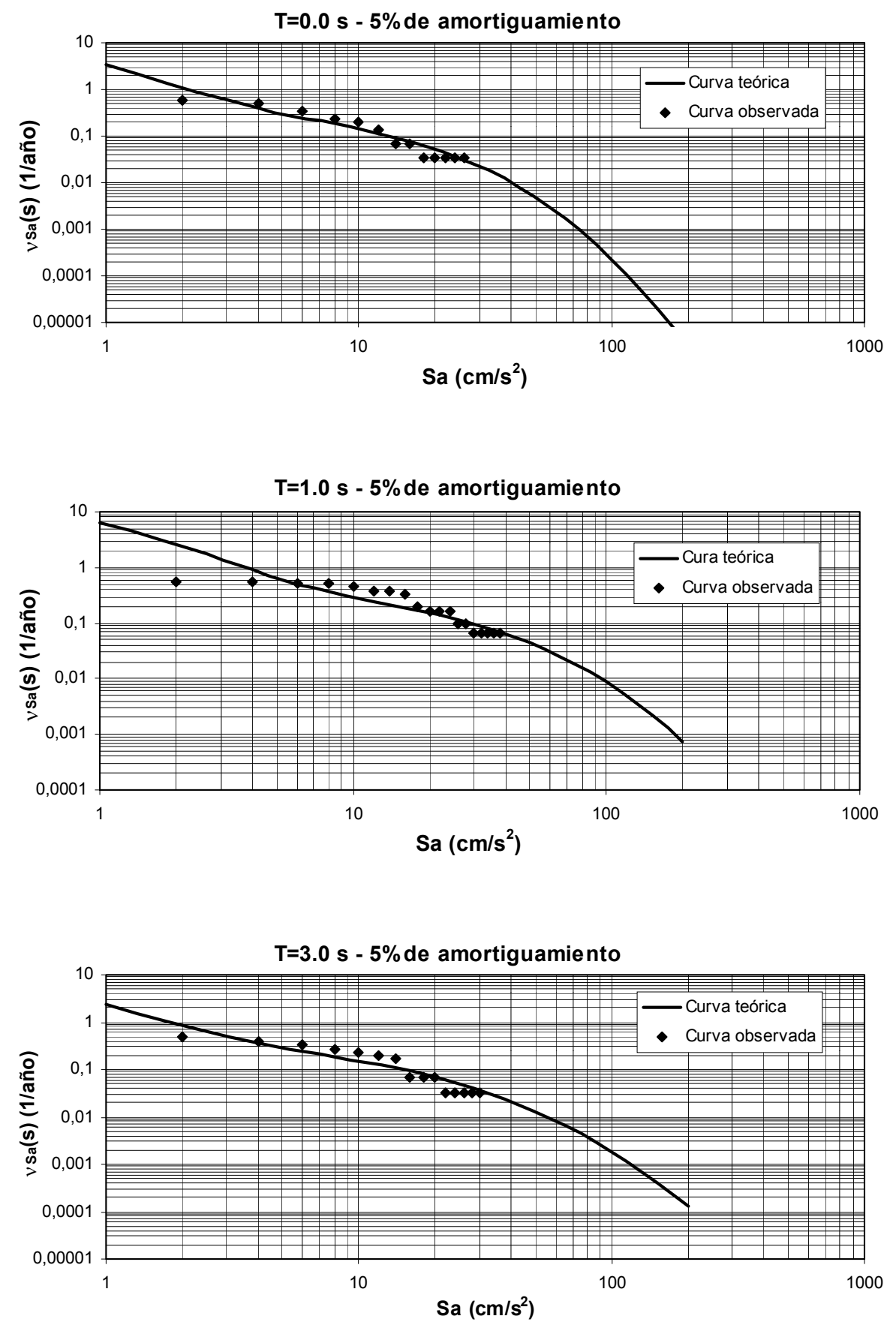

Figura 13. Curvas de peligro sísmico teóricas y observadas para la estación CU componente EW.

- En la parte central, las curvas observadas y teóricas se ajustan adecuadamente, lo que implica que el modelo matemático que se considera es el apropiado para esos niveles de aceleración;

- Para aceleraciones espectrales de baja intensidad (menores de $3 \mathrm{~cm} / \mathrm{s}^{2}$ ), la diferencia entre la curva calculada y la observada posiblemente esté asociada a la precisión de los aparatos de registro o bien al modelo matemático que se consideró; 
- En el caso de aceleraciones espectrales intensas, la diferencia entre la curva calculada y observada se puede asociar a que se cuenta con un número reducido de sismos que han generado este tipo de aceleraciones espectrales;

- La congruencia entre las curvas observadas y teóricas también implica que, en promedio, la ley de atenuación es adecuada para estimar espectros de respuesta para temblores futuros.

Cabe mencionar que la estación CU, es el único sitio en México y probablemente en el mundo donde existe suficiente información registrada para poder comparar las curvas de peligro sísmico calculadas con las observadas.

Construidas las curvas de peligro sísmico para cada periodo, si se desea un espectro para una tasa de excedencia o periodo de retorno seleccionado basta con leer de cada curva la ordenada espectral correspondiente. A los espectros construidos de esta manera se les conoce como espectros de peligro uniforme. En la figs. 14a, 14b y 14c se muestran este tipo de espectros construidos para la media geométrica de la aceleración espectral de los dos componentes del movimiento horizontal del terreno registrados en las estaciones 31, 36, 49, 51, 56 y SCT de la zona del lago, 27 de la zona de transición y la estación CU. Se puede observar que existen estaciones en la zona del lago que presentan niveles de amplificación del movimiento del terreno superiores al de la estación SCT. Esta estación se usa como referencia ya que fue una de las pocas estaciones que registraron el gran sismo del 19 de septiembre de 1985 en la zona del lago, y que hasta esa fecha era la evidencia de la amplificación más grande del movimiento del terreno en dicha zona.

\section{CONCLUSIONES}

En el presente trabajo se analizan dos procedimientos para estimar espectros de aceleraciones en la estación $\mathrm{CU}$, uno con el uso de una forma espectral normalizada y otro que emplea una ley de atenuación espectral. Al comparar ambos métodos, se concluye que con la ley de atenuación espectral se obtiene un menor error en las estimaciones, además de tener en cuenta la variación del espectro de respuesta con la magnitud; efecto que tiene gran importancia al estimar el espectro de respuesta en las zonas de terreno blando de la Ciudad de México usando los CER.

En una segunda parte, se estudia un procedimiento que emplea los CER para estimar el espectro de respuesta en estaciones de las zonas de transición y del lago de la ciudad de México. Del estudio se concluye que al estimar el espectro de respuesta con los CER, se obtienen resultados aceptables; esta conclusión coincide con los resultados de Arciniega et al. (1993). Sin embargo, también se observó que una fuente importante de incertidumbre es el procedimiento con el que se estima el espectro de respuesta en la estación CU. Por tanto, entre mayor sea la confiabilidad con que se estima el espectro de aceleraciones en la estación CU, mejor será la estimación del espectro en la zona de terreno blando de la ciudad de México.

En seguida se construyen, con base en la ley de atenuación espectral presentada en este trabajo, curvas teóricas de peligro sísmico en la estación $\mathrm{CU}$, éstas se comparan con las que se obtienen de los datos registrados en la misma estación. De la comparación se concluye que las 
curvas teóricas son congruentes con las observadas para intervalos de aceleraciones espectrales donde se puede realizar una comparación confiable, lo que implica que, en promedio, la ley de atenuación espectral estima de manera adecuada el espectro de aceleraciones en la estación CU.
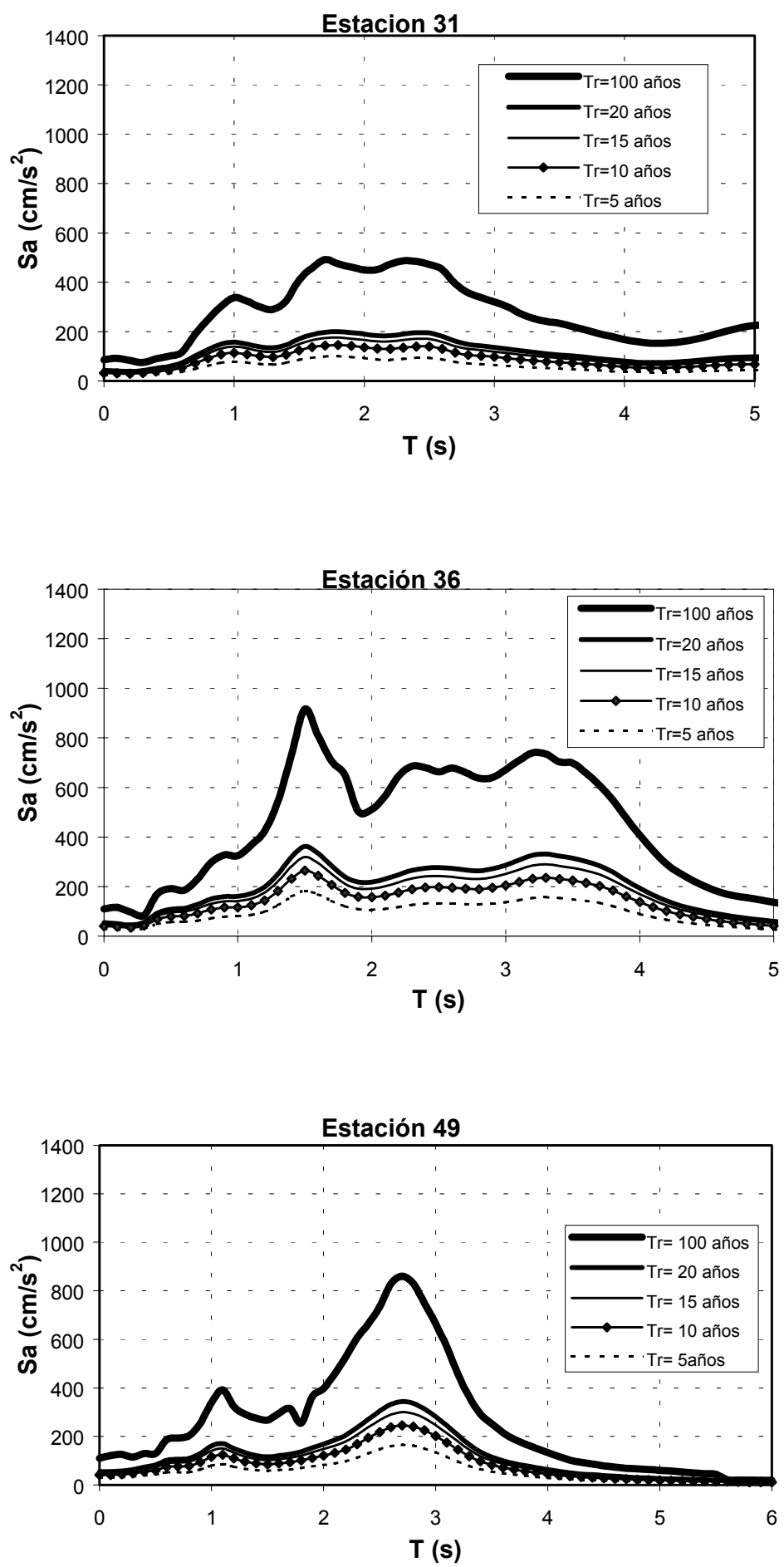

Figura 14a. Espectros para 5\% de amortiguamiento de peligro uniforme para distintos periodos de retorno considerando la media geométrica de la aceleración espectral. 

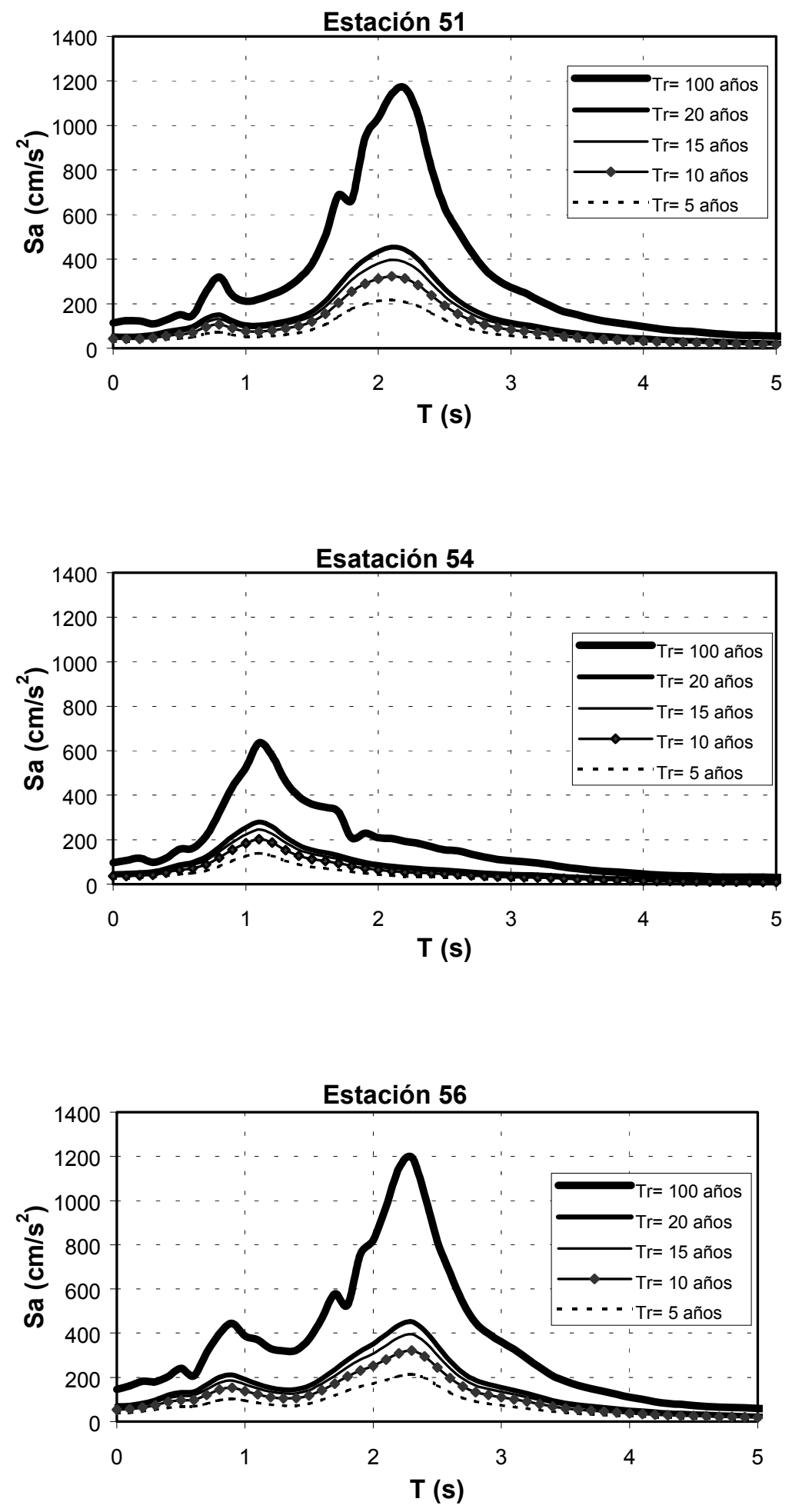

Figura 14b. Espectros para 5\% de amortiguamiento de peligro uniforme para distintos periodos de retorno considerando la media geométrica de la aceleración espectral. 

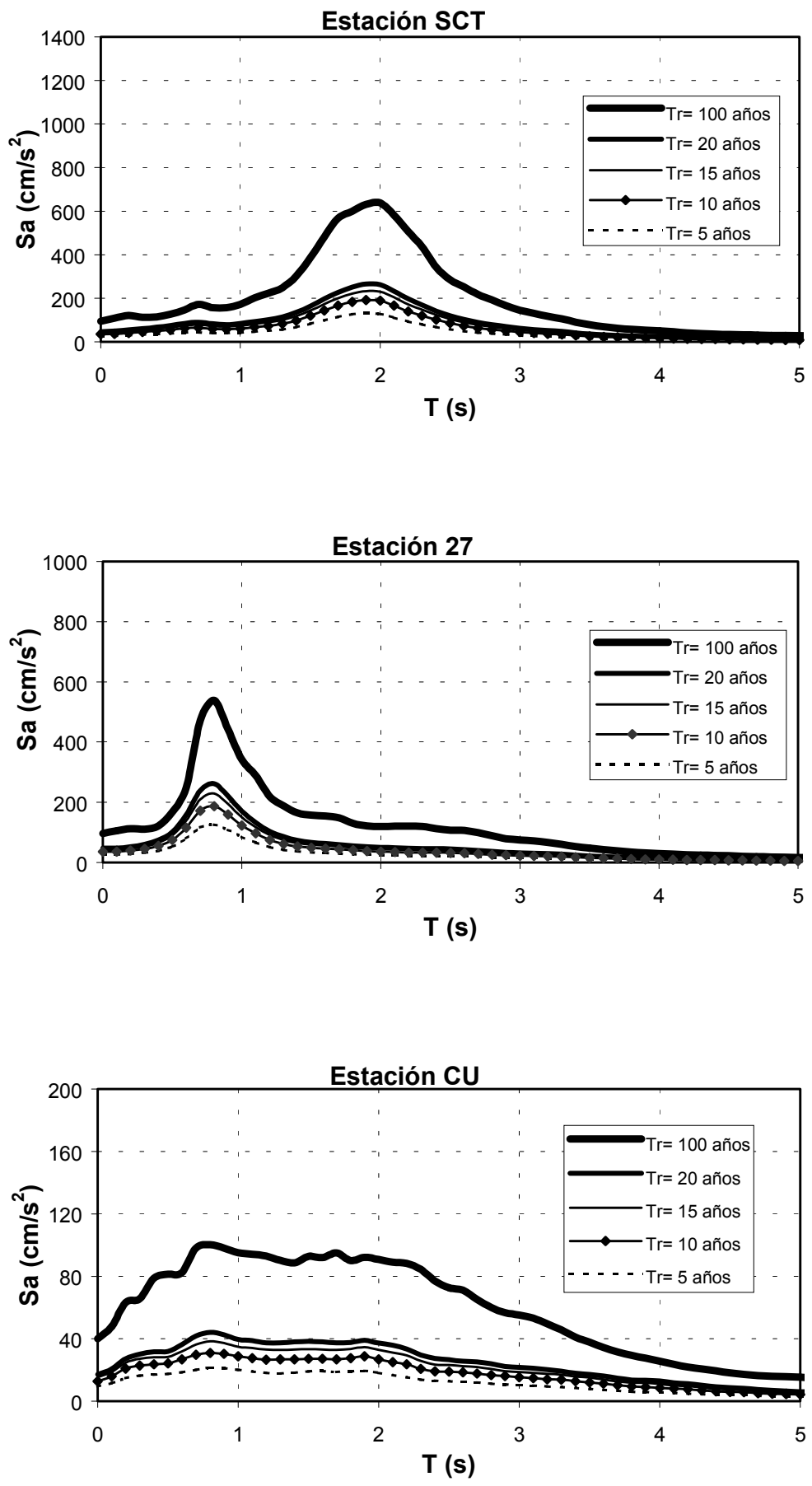

Figura 14c. Espectros de 5\% de amortiguamiento de peligro uniforme para distintos periodos de retorno considerando la media geométrica de la aceleración espectral.

Finalmente, se construyen espectros de peligro uniforme en la estación CU y en las estaciones localizadas en suelo blando. En las estaciones de suelo blando, los espectros de peligro uniforme se construyen mediante el uso de los CER. Se pudo observar que existen estaciones de registro sísmico que presentan amplificaciones superiores del movimiento del terreno en 
comparación con la estación SCT; recuérdese que esta estación fue la que registró el movimiento más intenso durante el sismo del 19 de septiembre de 1985.

\section{RECONOCIMIENTOS}

Se agradece al Centro de Instrumentación y Registro Sísmico y al Instituto de Ingeniería de la UNAM el haber proporcionado los registros sísmicos que se usaron en el presente estudio. Al Instituto de Ingeniería de la UNAM, también se le reconoce su apoyo, a través de un proyecto interno, para el desarrollo de este trabajo.

\section{REFERENCIAS}

Arciniega, A, M Ordaz, G Padilla, L E Pérez Rocha y E Rosenblueth (1993), "Predicción de espectros de respuesta en el valle de México. Métodos simplificados y aplicaciones," Reporte FJBS/CIS-93/04, Centro de Investigación Sísmica A.C., Fundación Javier Barros Sierra.

Benioff, H (1934), "The physical evaluation on seismic destructiveness," Bulletin of the Seismological Society of America, Vol. 24, No.2, pp. 398-403.

Biot, M A (1941), "A mechanical analyzer for the prediction of earthquake stresses," Bulletin of the Seismological Society of America, Vol. 31 No. 2, pp. 151-171.

Bufaliza, M (1984), "Atenuación de intensidades sísmicas con la distancia en sismos mexicanos", Tesis de maestría, Facultad de Ingeniería, Universidad Nacional Autónoma de México.

Cartwrigth, D E y M S Longuet-Higgins (1956), "The statistical distribution of the maxima of a random function," Memorias, Royal Society of London, Vol. A 237, pp. 212-232.

Davenport, A G (1964), "Note on the distribution of the largest value of a random function with application to gust-loading," Proc. Inst. Civil Eng., Vol. 28, pp.187-196.

Esteva, L (1968), "Bases para la formulación de decisiones en diseño sísmico," Publicación No. 182, Instituto de Ingeniería, Universidad Nacional Autónoma de México.

Esteva, L y R Villaverde (1974), "Seismic risk, design spectra and structural reliability," Memorias, Fourth World Conference on Earthquake Engineering, Rome Italy, pp. 2586-2597.

Housner, G W (1941), "An investigation of the effects of earthquakes on buildings," $P h$. $D$. Thesis, California Institute of Technology, Pasadena.

Housner, G W (1959), "Behavior of structures during earthquakes," Memorias, ASCE, Vol. 9, No.85, No.4, pp. 109-129.

Hayashi, S, H Tsuchida y E Kurata (1971), "Average response spectra for various subsoil conditions," Third Joint Meeting, U.S-Japan Panel of Wind and Seismic Effects, Tokio, Japón.

Joyner, W B y D M Boore (1988), "Measurement, characterization, and prediction of strong ground motion," Memorias, Earthquake Engineering and Soil Dynamics, II GT Div./ASCE, Park City, Utah, pp. 43-101, junio. 
Kanai, K (1961), “An empirical formula for the spectrum of strong earthquake motions”, Bull. Eq. Res. Inst., No.39, pp. 85-95.

McGuire, R K (1974), "Seismic structural response risk analysis, incorporating peak response regressions on earthquake magnitude and distance," Research Report R74-51, Dept. Civil Eng., Mass. Inst. of Tech., Cambridge, Mass.

Ordaz, M y E Reinoso (1987), "Uso de la teoría de vibraciones aleatorias en la determinación de los espectros de diseño del reglamento para las construcciones del Distrito Federal," Memorias del VII Congreso Nacional de Ingeniería Sísmica, Querétaro Qro.,México, pp. A155-A167.

Ordaz, M, J M Jara y S K Singh (1989), "Riesgo sísmico y espectros de diseño para el estado de Guerrero," Informe interno 8782/9745 del Instituto de Ingeniería de la Universidad Nacional Autónoma de México y la Fundación Javier Barros Sierra A.C.

Ordaz, M y S K Singh (1992), "Source spectra and spectral attenuation of seismic waves from Mexican earthquakes and evidence of amplification in the hill zone of Mexico City," Bulletin of the Seismological Society of America, Vol. 82, No.1, pp. 24-43.

Ordaz, M, S K Singh y A Arciniega (1994), "Bayesian attenuation regressions: an application to Mexico City,” Geophysics Journal International, Vol. 117, pp. 335-344.

Ordaz, M y C Reyes (1999), "Earthquake hazard in Mexico City: observations versus computations,'" Bulletin of the Seismological Society of America, Vol. 89, No.5, pp. 1379-1383.

Perea, T y E Sordo (1998), "Direct response spectrum prediction including local site effects," Memoria de la décimo primera conferencia europea sobre ingeniería sísmica, París, Francia, en disco compacto.

Reyes C (1999), "El estado límite de servicio en el diseño sísmico de edificios," Tesis Doctoral, División de Estudios de Posgrado de la Facultad de Ingeniería de la Universidad Nacional Autónoma de México.

Rosenblueth, E y A Arciniega (1992), "Response spectral ratios," Earthquake Engineering and Structural Dynamics, Vol. 21, pp. 483-492.

Seed, H B, C Ugas y J Lysmer (1976), "Site-dependent spectra for earthquake resistant design," Bulletin of the Seismological Society of America, Vol. 66, No. 1, pp. 221-243.

Singh, S K, M Rodríguez y J M Espíndola (1984), "A catalog of shallow earthquakes of Mexico from 1900 to 1981," Bulletin of the Seismological Society of America, Vol. 74, No.1, pp. 267-279.

Singh, S K, E Mena y E Castro (1988), "Prediction of peak, horizontal ground motion parameters in Mexico City from coastal earthquakes", Geofisica Internacional, Vol. 27, No. 1, pp. 111-129.

Trifunac, M y J G Anderson (1978), "Preliminary empirical models for scaling pseudo relative velocity spectra," Appendix A in Methods for Prediction of Strong Earthquake Ground Motion, U.S. Nuclear Regulatory Commission Report NUREG/CR-0689, A1-A90.

Udwadia, F E y M D Trifunac (1974), "Characterization of response spectra trough the statistic of oscillator response," Bulletin of the Seismological Society of America, Vol. 64, No.1, pp. 205219.

Zúñiga, R (1996). Comunicación personal. 